\title{
A Systematic Review of Biopsychosocial Training Programs for the Self-Management of Emotional Stress: Potential Applications for the Military
}

\author{
Cindy Crawford, ${ }^{1}$ Dawn B. Wallerstedt, ${ }^{1}$ Raheleh Khorsan, ${ }^{2}$ Shawn S. Clausen, ${ }^{3}$ \\ Wayne B. Jonas, ${ }^{1}$ and Joan A. G. Walter ${ }^{1}$ \\ ${ }^{1}$ Samueli Institute, 1737 King Street, Suite 600, Alexandria, VA 22314, USA \\ ${ }^{2}$ Samueli Institute, 2101 East Coast Highway, Suite 300, Corona Del Mar, CA 92625, USA \\ ${ }^{3}$ Walter Reed National Military Medical Center, 8901 Wisconsin Avenue, Building 8, Room 5106, Bethesda, MD 20889, USA \\ Correspondence should be addressed to Cindy Crawford; ccrawford@siib.org
}

Received 10 May 2013; Revised 26 June 2013; Accepted 22 July 2013

Academic Editor: Tobias Esch

Copyright ( 92013 Cindy Crawford et al. This is an open access article distributed under the Creative Commons Attribution License, which permits unrestricted use, distribution, and reproduction in any medium, provided the original work is properly cited.

Combat-exposed troops and their family members are at risk for stress reactions and related disorders. Multimodal biopsychosocial training programs incorporating complementary and alternative self-management techniques have the potential to reduce stressrelated symptoms and dysfunction. Such training can preempt or attenuate the posttraumatic stress response and may be effectively incorporated into the training cycle for deploying and redeploying troops and their families. A large systematic review was conducted to survey the literature on multimodal training programs for the self-management of emotional stress. This report is an overview of the randomized controlled trials (RCTs) identified in this systematic review. Select programs such as mindfulnessBased Stress Reduction, Cognitive Behavioral Stress Management, Autogenic Training, Relaxation Response Training, and other meditation and mind-body skills practices are highlighted, and the feasibility of their implementation within military settings is addressed.

\section{Introduction}

Combat-exposed troops and their family members are at risk for stress reactions and related disorders [1]. Strategies to enhance psychological resilience among service members are needed. Providing this training prior to deployment might preempt or attenuate the posttraumatic stress response, depression, anxiety, and other consequences of overwhelming stress.

Complementary and alternative medicine (CAM) and integrative medicine (IM) approaches to self-management of emotional stress are increasingly utilized within comprehensive care models [2]. Surveys have affirmed the widespread use of integrative modalities in military populations and settings, including Department of Defense (DoD) beneficiaries [3], active duty military [4], and patients using Veterans Health Administration (VHA) hospitals [5-7].
Multimodal treatment programs, as compared to single modality treatments, have emerged as an important option in the management of stress disorders $[8,9]$. Compared to treatment with a single modality, multimodal programs have the potential to simultaneously address a range of stress reactions, both physical and mental, as well as the dynamic nature of the disease process over time. Applied at the population level, the increased variety of modalities potentially has a greater chance of providing viable alternatives for a given individual.

The military is already a culture in which self-care is recognized as a vital tool in warfare: adequate nutrition, hydration, and sleep are part of a warrior's battle kit. Therefore, selfmanagement skills that are delivered as multimodal programs involving CAM/IM may be an ideal option for the military community to help build resilience, reset the autonomic 
nervous system, and ease emotional stress. Consequently, in tandem with the mainstreaming of many CAM practices in the civilian sector, military personnel may seek CAM therapies to improve their health and well-being, especially those CAM therapies that are self-manageable and drugless.

A number of model biopsychosocial training programs exist in the civilian sector [10-13]. Several have adapted their programs to the military $[10,12-14]$, and a smaller number have provided training to military personnel [14]. However, to date, there has been no comprehensive systematic review of multimodal biopsychosocial programs for the self-management of stress. The authors posit that these interventions could be markedly effective for individuals in high-stress environments such as the military.

1.1. Purpose of the Review. A comprehensive systematic review was conducted to (1) survey multimodal biopsychosocial training programs with at least one CAM/IM component for the self-management of emotional stress across all populations; (2) assess the quantity and quality of the research and programs; and (3) characterize the results by type of program and strength of evidence on stressrelated outcome measures. Due to the massive amount of the literature included, the full report of this review will be detailed in a much larger report, not suitable for detailing in one paper. The purpose of this current report is to (1) focus on those studies that were scored as high quality and demonstrated statistically significant results between groups (i.e., intervention group versus control or comparison group) on outcomes of emotional stress for controlled trial study designs; (2) describe the multimodal programs available and their characteristics; (3) describe the results of outcomes related to emotional stress; and (4) discuss what the authors believe to be the resource requirements needed to incorporate these programs into a military setting for service members and their families. The authors have chosen to report this subset of data since studies assessed as high quality according to internal validity criteria are the least likely to have introduced bias, are more likely replicable, and can be trusted to show a valid effect for the intervention and population being studied $[15,16]$. If these studies are generalizable to other populations, then it is reasonable to anticipate that an effective program, if implemented in a military environment, could show equivalent benefits for this highly stressed population.

\section{Methods}

\subsection{Concepts and Definitions}

2.1.1. Biopsychosocial Model. The biopsychosocial model (abbreviated "BPS") is a term introduced in 1977 by the American psychiatrist George Engel which describes a health care perspective that acknowledges that biological, psychological (which entails thoughts, emotions, and behaviors), and social factors all play a significant role in human functioning in the context of wellness and illness. It is a term that is often used to describe the concept of the "mind-body connection" [8].
2.1.2. Complementary and Alternative Medicine (CAM) Modality. CAM is defined at the National Center for Complementary and Alternative Medicine as: any of a number of "diverse medical and health care systems, practices, and products that are not generally considered part of conventional medicine" (http://nccam.nih.gov/). For the purposes of this review, the authors included only CAM modalities that also met our criteria for the biopsychosocial model and self-management technique (http://www.ncbi.nlm.nih.gov/mesh/?term=complementary therapies) and used definitions of CAM techniques of breathing, relaxation, yoga, imagery, hypnosis, and meditation as described by the National Center for Health Statistics on the NCCAM website: http://nccam.nih.gov/ health/providers/camterms.htm.

2.1.3. Self-Management Technique. Self-management techniques are techniques in which skills are used independently by an individual without ongoing reliance on a trainer or therapist. The authors excluded interventions where patients solely learn and integrate therapies by themselves (such as through a book or online material) or ones that are trainer or therapist dependent (i.e., psychotherapy that requires a therapist to lead the sessions).

2.1.4. Multimodality Interventions. These interventions are defined as those ones that have two or more interventions (at least one of which is CAM modality) that require an initial training period with a therapist or trainer in which skills are learned, all of which can be transferred into self-management techniques. The authors only included programs that have multimodal interventions. An example is a program that includes relaxation, exercise, and behavioral techniques to reduce stress. The thought here was that multimodal programs would allow for more of a biopsychosocial approach to treating the whole person for the complexities of emotional stress.

2.1.5. Types of Program. Types of programs that the authors considered were those that include training in at least one self-management multimodal training with the intention to reduce psychological or emotional stress. The program did not necessarily have to be an existing named program per se but had to include interventions that could be developed into a program (e.g., a potential program). The authors included educational training programs as long as they met these criteria.

2.2. Search Strategy. The following electronic databases were searched from database inception through February 2009 across keywords identified: PUBMED, EmBase, BIOSIS, CINAHL, the entire Cochrane library as well as the database of abstracts of reviews of effectiveness (DARE), PILOTS, PsycInfo, AMED, ERIC, and DoD Biomedical Research. Gray literature was also searched for unpublished trials via the Register of the Controlled Trials databases (http://www.controlled-trials.com/ and http://www.clinicaltrials.gov/), NLM catalog, and NCCAM 
Grantee Publications Database, communicating with identified experts in the field of CAM/IM for additional reports of studies not included through traditional searching and pearling references of included articles.

2.3. Study Selection. Studies were included if they involved (1) research on a program or potential program; (2) a multimodal intervention incorporating at least one CAM modality (as defined by NCCAM and detailed by the National Library of Medicine (NLM) http://nccam.nih .gov/health/providers/camterms.htm; http://www.ncbi.nlm .nih.gov/mesh/?term=complementary+therapies); (3) skills that were learned that could be used as self-management techniques, after a training period; (4) at least one outcome measure of psychological or emotional stress; (5) human subjects from any clinical or nonclinical population; and (6) were presented in the English language. To encompass the construct of "psychological or emotional stress," studies were included that used outcome measures containing one of the following keywords: stress, anxiety, post-traumatic stress disorder, coping, resilience, hardiness, burnout, distress, or relaxation at the screening phase. Since all healthy and clinical populations experience stress (although to varying degrees), the authors elected not to exclude any population based on predefined criteria about their conditions or diagnoses. This was consistent with the authors' intent to generalize about the value of these programs impacting the symptoms of stress. Thus, all populations, both healthy and clinical (e.g., those with specific conditions or diagnoses), were included as long as the report included a description of emotional stress as defined above. Types of the literature excluded from this systematic review, were thought pieces, descriptive reviews or published expert opinions. The authors excluded the following interventions: ones in which individuals learned and integrated therapies by themselves; those that involved website training, books, or leaflets as the sole source of the training; pharmacological agents or placebos; and pet therapy. Cognitive behavioral therapy (CBT) was not defined as a CAM practice by itself but was included when it incorporated another CAM technique, such as deep breathing or relaxation exercises that were the predominant feature of the program. All programs had to involve at least one CAM modality as described above; no additional medical or psychosocial procedures were included unless the program integrated those procedures with the CAM modality for the purpose of stress management. See Box 1 for the search terms used.

Five investigators (Cindy Crawford, Sasha Knowlton, Raheleh Khorsan, Dawn Wallerstedt, and Shawn Clausen) individually and independently screened all titles and abstracts in duplicate for relevance based on the inclusion criteria mentioned above. Weekly team meetings were held between all five screeners to resolve any and all disagreements.

2.3.1. Quality Assessment and Data Extraction. The methodological quality of included RCT studies was assessed independently by four reviewers in duplicate using a modified version of the Scottish Intercollegiate Guidelines Network (SIGN 50) checklist, a validated and reliable assessment approach widely used in the literature [17]. Three of the 10 SIGN criteria for assessment of quality were omitted as they did not apply to our research question (see Table 1). High quality was defined as a SIGN 50 score equal to + (only 1-2 criteria scored as poorly addressed $)$ or $++(0$ criteria scored as poorly addressed); that is, some or all of the internal validity criteria have been fulfilled. Where they have not been fulfilled, the conclusions of the study were thought unlikely to very unlikely to alter results [17]. All reviewers were fully trained in the methodology employed. All conflicts were resolved through discussion and consensus or by consulting the senior author. Samueli Institute developed a rulebook to ensure objectivity in scoring and reliability between reviewers to improve the often subjective assessments in quality criteria scoring in systematic reviews. As detailed above and in Table 1, the individual criteria were "weighted" to account for the omission of criteria that did not apply to this body of literature.

2.4. Results. The initial search from the full systematic review yielded 11,977 citations from database inception through February 2009, of which 284 reports were deemed suitable to be included, with 116 being RCT study designs. See Figure 1 for the flow diagram of studies throughout the review phases. This current report includes only those that were RCT's and were scored as high quality and statistically significant results. Subsequent planned reports will comment on other study designs available.

2.5. Types of Programs. The 116 RCT studies from the full systematic review were categorized into the following types of programs: those that have been previously characterized/named (56 total): Mindfulness-Based Stress Reduction (MBSR), Cognitive Behavioral Stress Management (CBSM), Autogenic Training (AT), Relaxation Response Training (RRT), Stress Inoculation Training (SIT), Anxiety Management Training (AMT), and Coping Skills Training (CST); and those that have not been previously characterized/named (60 total): yoga and similar meditation-based modalities (including programs that incorporated yoga-type techniques as the primary intervention) and relaxation and other similar mind-body skills (including programs that used any relaxation technique, breathing, guided imagery, self-hypnosis, and/or Cognitive Behavioral Therapy (CBT) as the primary intervention). Table 2 displays the number of RCT studies, categorized by name of program, quality rating (SIGN 50 score), and significance level showing between-group differences on stress-related outcome results. Of note, very few of the high-quality studies reported negative results. None of the studies that used CST, AMT, or SIT as an intervention fit the criteria of high quality; therefore, these will not be reported on further in this report but will be described in subsequent publications. Detailed descriptions of each of the 34 studies that were of high quality and yielded statistically significant results between groups are displayed in Table 3 and described below. Because the unnamed programs' 
(program OR programme OR training OR taught OR learn* OR skill*) AND (stress OR “Stress, Physiological” [Mesh] OR "Stress Disorders, Post-Traumatic" [Mesh] OR "Stress, Psychological” [Mesh] OR "Stress Disorders, Traumatic" [Mesh]) AND (psychosocial OR integrat* OR "alternative medicine" OR "complementary medicine" OR "alternative therapy" OR "complementary therapy" OR "complementary and alternative medicine" OR "complementary and alternative therapy" OR mind-body OR imagery OR mindfulness OR yoga OR yogic OR biofeedback OR "heart-rate variability" OR "virtual reality" OR breath* OR "cognitive restructuring" OR mantr* OR acupressure OR "body scan" OR "autogenic training" OR self-care OR self-awareness OR self-management OR Reiki OR massage OR "therapeutic touch" OR holistic OR wholistic OR prayer OR writing OR "movement therapy" OR "dance therapy" OR "narrative medicine" OR self-acupuncture OR qigong OR chi OR qi OR "spiritual healing" OR meditat* OR "mental training” OR self-hypnosis OR relax* OR EMDR)

Box 1: Search terms used according to MeSH strategy.

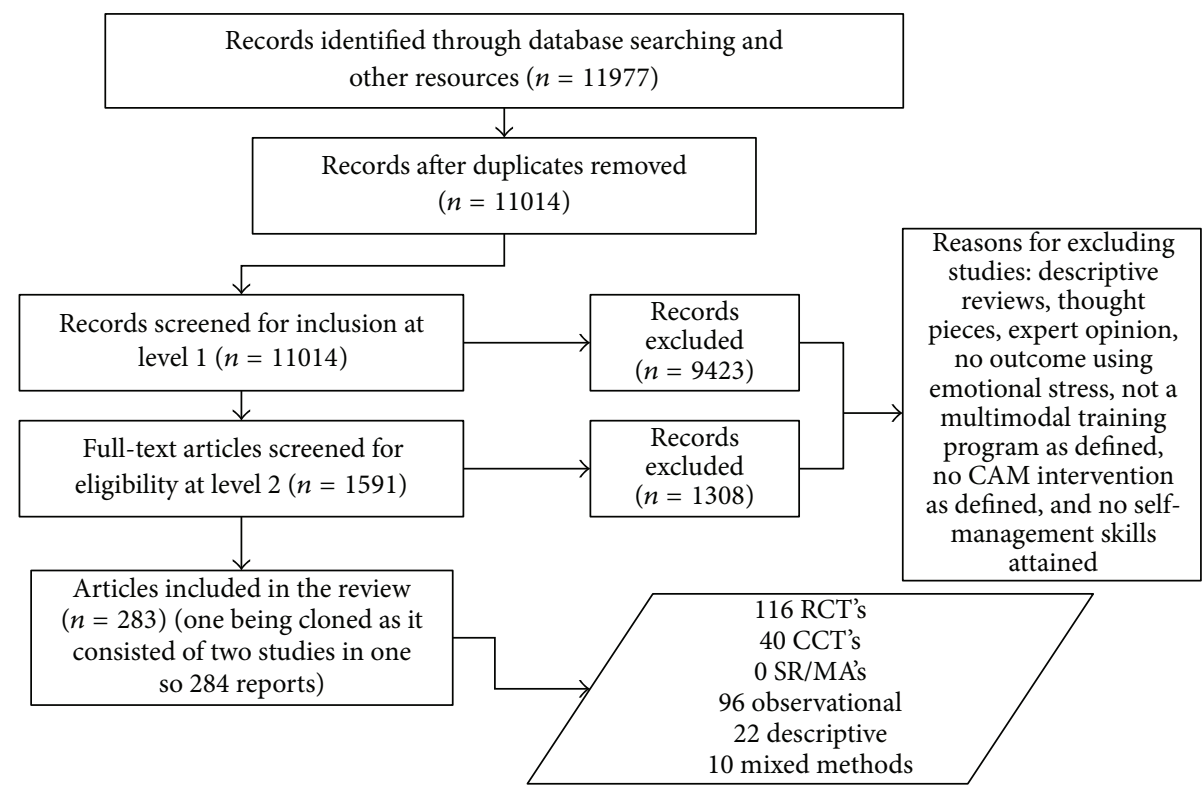

FIGURE 1: Flowchart of study selection process.

content and heterogeneity varied across studies, the authors provide a full description of the program incorporated in each study in Table 3(b).

\subsection{Descriptive Overview of Included High-Quality Programs}

2.6.1. Mindfulness-Based Stress Reduction (MBSR). Mindfulness-Based Stress Reduction was developed approximately 30 years ago by Dr. Jon Kabat-Zinn and now has evolved into a structured group program (http://www .umassmed.edu/cfm/stress/index.aspx). It uses meditation as a tool to cultivate conscious awareness in a nonjudgmental and accepting manner. MBSR has been used to help individuals with stress, chronic pain, anxiety, sleep, and headache, among others $[18,19]$. The MBSR course schedule generally consists of eight weekly classes and one day-long retreat, including guided instruction on mindfulness meditation practices, gentle stretching and mindful yoga, group dialogue and discussions aimed at enhancing awareness in everyday life, individually tailored instruction, daily home assignments, and home practice CDs.
2.6.2. Cognitive Behavioral Stress Management (CBSM). Cognitive Behavioral Stress Management is a multimodal program adapted from a variety of meditation and cognitive behavioral strategies and has been used for more than 20 years by a variety of groups. CBSM has been used to help individuals with coping, quality of life, psychological well-being, PTSD, and HIV-related stressors [20]. CBSM is generally a ten-week group-based program that combines relaxation, imagery, and deep breathing, along with cognitive behavior therapy, which is designed to help reduce bodily tension, intrusive stressful thoughts, and negative moods and improve interpersonal communication skills [21].

2.6.3. Autogenic Training (AT). Autogenic Training was developed by the German psychiatrist Johannes Schultz in 1932. The goal of AT is to achieve deep relaxation and reduce stress by teaching the body to respond to verbal commands "telling" it to relax and control breathing, blood pressure, heartbeat, and body temperature [22]. It includes standardized self-suggestion exercises to make the body feel warm, heavy, and relaxed [23-26]. 
TABle 1: Modified SIGN 50 checklist for RCT study design [17].

(a) Section 1: internal validity*

\begin{tabular}{ll}
\hline Item & Description \\
\hline 1.1 & The study addresses appropriate and clearly focused question. \\
1.2 & The assignment of subjects to treatment groups is randomized. \\
1.5 & The treatment and control groups are similar at the start of the trial. \\
1.7 & All relevant outcomes are measured in a standard, valid and reliable way. \\
1.8 & What percentage of subjects in each treatment arm dropped out before the study was completed? \\
1.9 & All subjects are analyzed in the groups to which they were randomly allocated (intention to treat analysis). \\
1.10 & Where the study is carried out at more than one site, results are comparable for all sites. \\
\hline
\end{tabular}

Each item in Section 1 is to be evaluated using these criteria: well covered; adequately addressed; poorly addressed; and not applicable (NA) only for question 1.10 .

${ }^{*}$ Note that 1.3, 1.4, and 1.6 SIGN criteria were omitted from our modified version of the SIGN as they did not apply to our research question/population: as there were wide differences in the types of programs assessed. Note that all criteria were weighted according to a revised SIGN quality score as reflected below consistently.

(b) Section 2: overall assessment

\begin{tabular}{l}
\hline $\begin{array}{l}\text { How well was the study done to minimize bias? How valid is the study? Score options: }++,+ \text {, and - based on the following } \\
\text { (modifications to SIGN criteria in italics). }\end{array}$ \\
\hline$+\quad \begin{array}{l}\text { All or most of the criteria have been fulfilled. Where they have not been fulfilled, the conclusions of the study are thought } \\
\text { "very unlikely" to alter. An article receives this score if there are } 0 \text { criteria scored as poorly addressed. }\end{array}$ \\
$+\quad \begin{array}{l}\text { Some of the criteria have been fulfilled. Those criteria that have not been fulfilled or not adequately described are thought } \\
\text { "unlikely" to alter the conclusions. An article receives this score if } 1-2 \text { criteria are scored poorly addressed. }\end{array}$ \\
Few or no criteria fulfilled. The conclusions of the study are thought "likely or very likely" to alter. An article receives this \\
score if more than 2 criteria are scored as poorly addressed.
\end{tabular}

TABLE 2: Randomized controlled trials $(n=116)$ by SIGN score and significance level ${ }^{*}$.

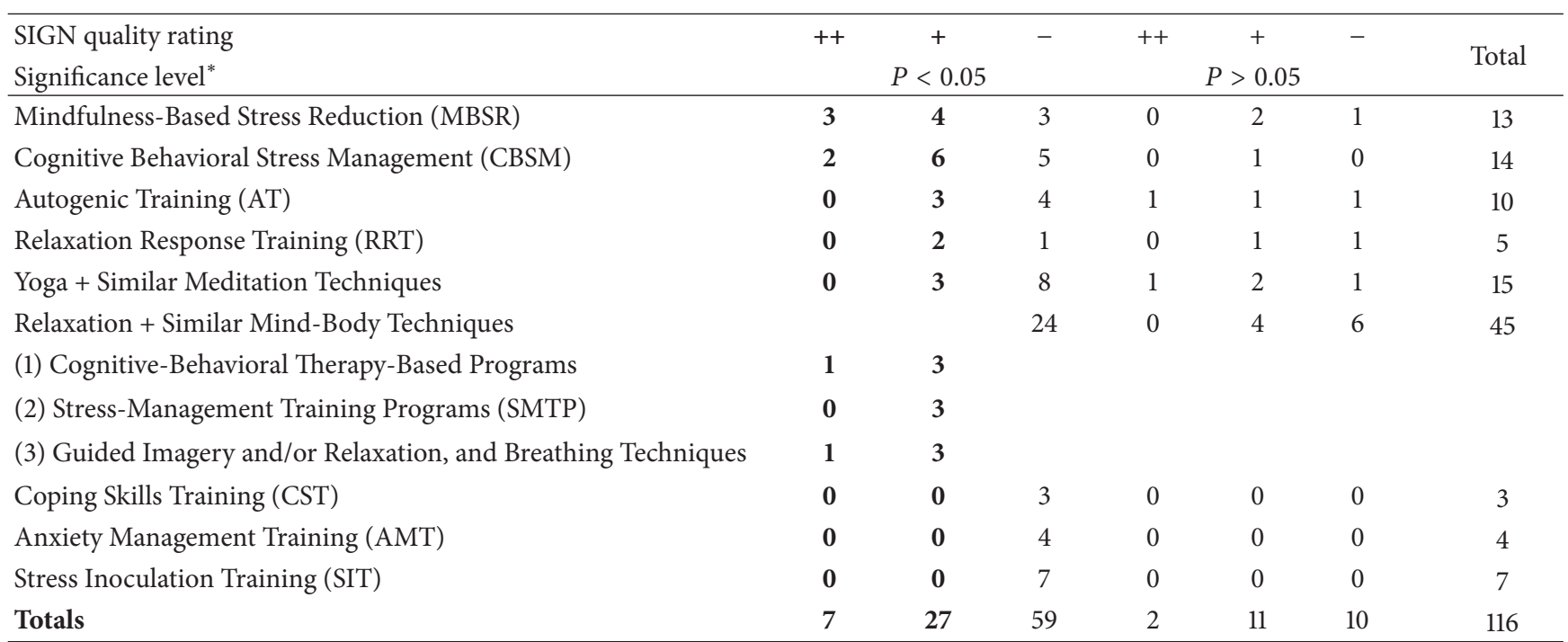

* On stress-related outcomes with keywords: stress, anxiety, post-traumatic stress disorder, coping, resilience, hardiness, burnout, distress, or relaxation.

2.6.4. Relaxation Response Training (RRT). Relaxation Response Training is a stress-management approach first published in 1974 by the cardiologist Benson et al. [27]. Benson found that meditation was related to general reversal of the sympathetic activation that produces the "stress response" (i.e., decreased oxygen consumption, carbon dioxide production, respiratory rate, and minute ventilation)
[28]. RRT was originally based on transcendental meditation but differentiated into its own technique using the following four elements to elicit the relaxation response: (1) a mental device (e.g., a sound, word, or phrase repeated silently or audibly to free one's self from logical, externally oriented thought); (2) a passive attitude (e.g., not worrying about how well one is performing the technique); (3) a decreased 
muscle tonus (e.g., comfortable, relaxed posture); and (4) a quiet environment with minimal environmental stimuli (e.g., a place of worship) [29].

2.6.5. Yoga + Similar Meditation-Based Skills. This miscellaneous category included studies that were not "named programs" and did not fit into any of the previously characterized categories and so were compiled together as a single, distinct category. These interventions met the inclusion criteria as programs that incorporated at least one yoga-based or meditation-based element as primary intervention. See Table 3(b) for a more complete description of each of these programs.

2.6.6. Relaxation + Similar Mind-Body Skills. Another miscellaneous category also included studies of programs that did not fit into any of the previously named categories. These incorporated at least one relaxation technique (such as progressive muscle relaxation) combined with other modalities such as breathing, guided imagery, and/or CognitiveBehavioral Therapy (CBT). While all of these studies had in common that they integrated at least one relaxation technique, for ease of discussion, the authors have grouped them into three subcategories: those that were based on a CBT model, those that were characterized as "StressManagement Training Programs," and those that combined relaxation with either guided imagery or breathing techniques. Cognitive-behavioral therapy is a well-established and effective psychotherapy approach for conditions such as anxiety and depression [62, 63], which assist individuals to recognize distorted thoughts, devise strategies to reframe them, and change resultant reactions and behaviors (http://www.nacbt.org/). An example of a CBT program that incorporates a relaxation component includes sessions on understanding the nature of stress and stress reactions, breathing and/or relaxation techniques, and cognitive restructuring techniques (i.e., self-talk skills to use in stressful situations) [51].

Stress-Management Training Programs (SMTPs) incorporate a number of skills and techniques to assist individuals to attenuate their physiological and psychological reactivity to stressful situations, including problem-solving, assertiveness training, and coping skills with various relaxation techniques, such as breathing and Progressive Muscle Relaxation (PMR). In this review, a program was tagged as "SMTP" only if the intervention was described using this term.

Guided imagery (GI) is a relaxation technique that focuses on and directs the imagination to produce therapeutic change (http://www.healthjourneys.com/) and can be administered by a trained practitioner leading an individual or group session or delivered as a recording. GI frequently includes suggestions for breathing and relaxation, followed by a purposeful directing of the imaginal mind to recreate a relaxing scene with sensory recruitment to enhance a multisensory experience. See Table 3(b) for a more complete description of these programs.
2.7. Results of the High-Quality Studies Included. Of the 13 MBSR studies included, seven high-quality reports $(3++$ and $4+$ ) were found to produce statistically significant effects on outcomes of distress in 63 rheumatoid arthritis patients [30]; perceived stress in 47 undergraduate students [33]; anxiety and perceived stress in 109 cancer patients [34]; anxiety and distress in 78 premedical students [32]; anxiety in 20 heart disease patients [31]; distress in 104 premedical students [25]; and distress and perceived stress in 103 volunteers with high levels of perceived stress [26].

Of the 14 CBSM studies included, eight high-quality reports $(2++$ and $6+)$ were found to demonstrate statistically significant effects on outcomes of coping in $387 \mathrm{HIV}$ patients [35]; everyday life stress in 198 heart disease patients [36]; anxiety in $104 \mathrm{HIV}$ patients [37]; anxiety in 37 third semester economic students [38]; perceived stress in 48 university students [39]; coping and relaxation in 199 breast cancer patients [40]; anxiety in 199 breast cancer patients [41]; and coping in $52 \mathrm{HIV}$ patients [42].

Of the 10 AT studies included, three high-quality reports $(3+)$ were found to yield statistically significant effects on outcomes of anxiety in 93 nursing students with anxiety [44]; anxiety in 100 acute myocardial infarction or coronary artery bypass surgery patients [43]; and distress in 56 patients with chronic tension headache [45]. This final study included a combination program consisting of AT and self-hypnosis [45].

Of the five studies on RRT included, two high-quality reports $(2+)$ were found to have statistically significant outcomes of psychological distress in 128 healthy undergraduate and graduate students (a program involving RRT and CBT training) [46] and distress in 80 patients with psychosomatic complaints [47].

Included in the yoga or meditation-based category were three high-quality reports $(3+)$ that were statistically significant on measured outcomes of the study's reports. These three studies consisted of yoga, meditation and relaxation, breathing or imagery, or a combination of all, which measured perceived stress in 259 participants who had experienced a hurtful interpersonal experience from which they still felt negative emotional consequence [48] and two studies on women with breast cancer that were statistically significant on outcomes of trait anxiety stress plus state anxiety according to STAI $(n=34)$ [49] and an integrated yoga program $(n=98)$ [50].

In this relaxation-based category, 35 of 45 studies reported statistically significant differences in stress-related outcomes; $11(24 \%)$ were classified as high quality $(++$ or +$)$. Four studies examined CBT-based programs that incorporated relaxation techniques. In a study of 108 patients with severe mental illness and PTSD, an eight-session CBT program with breathing techniques resulted in significant improvements in trauma-related cognitions, anxiety, and PTSD symptoms [51]. In another study of 31 chronic schizophrenic inpatients, a 12-hour CBT program with breathing exercises resulted in significant reductions in work-related stress [53]. In a third study, a 60-hour CBT program with qi gong (a meditative breathing technique) and relaxation exercises resulted 


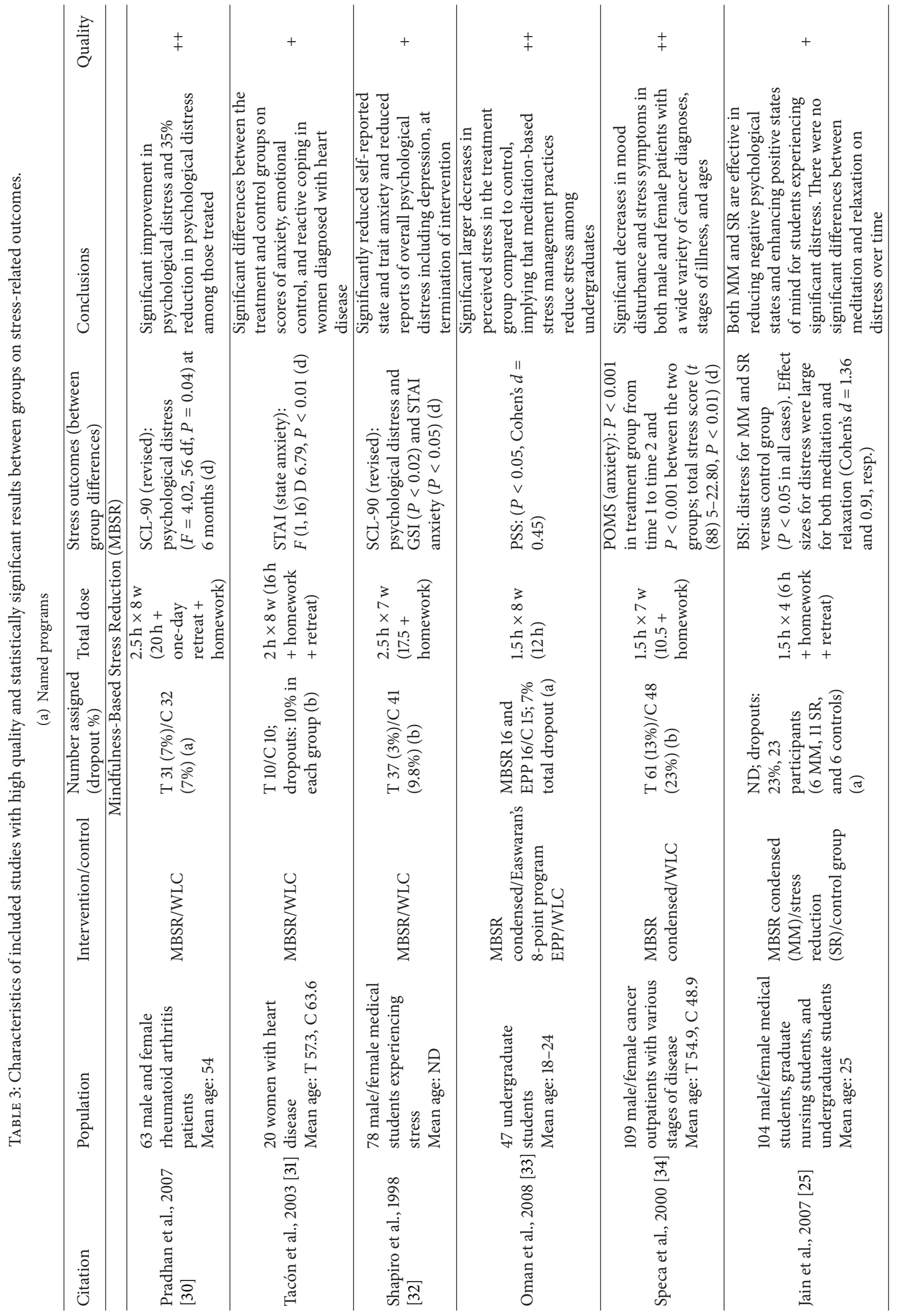




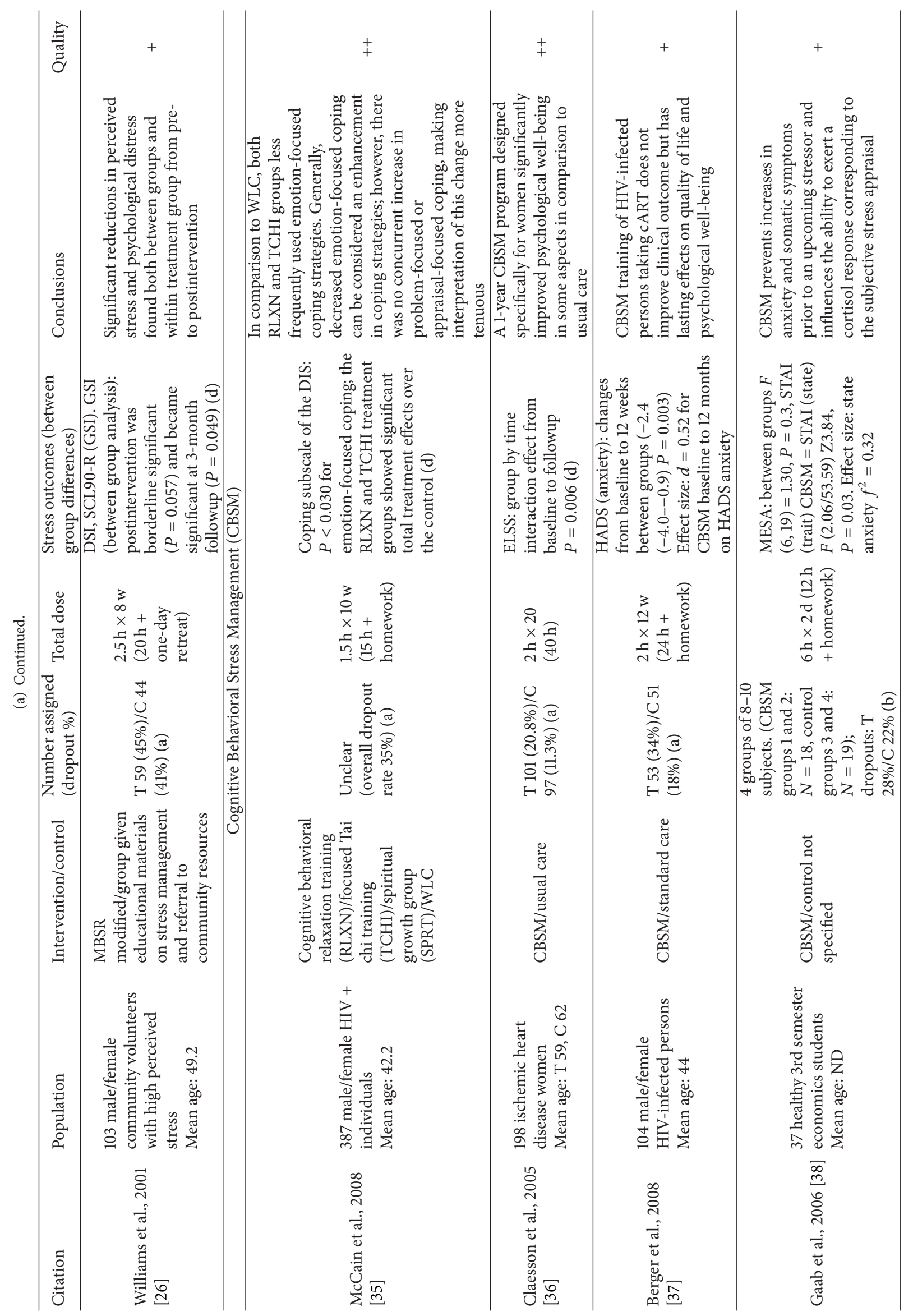




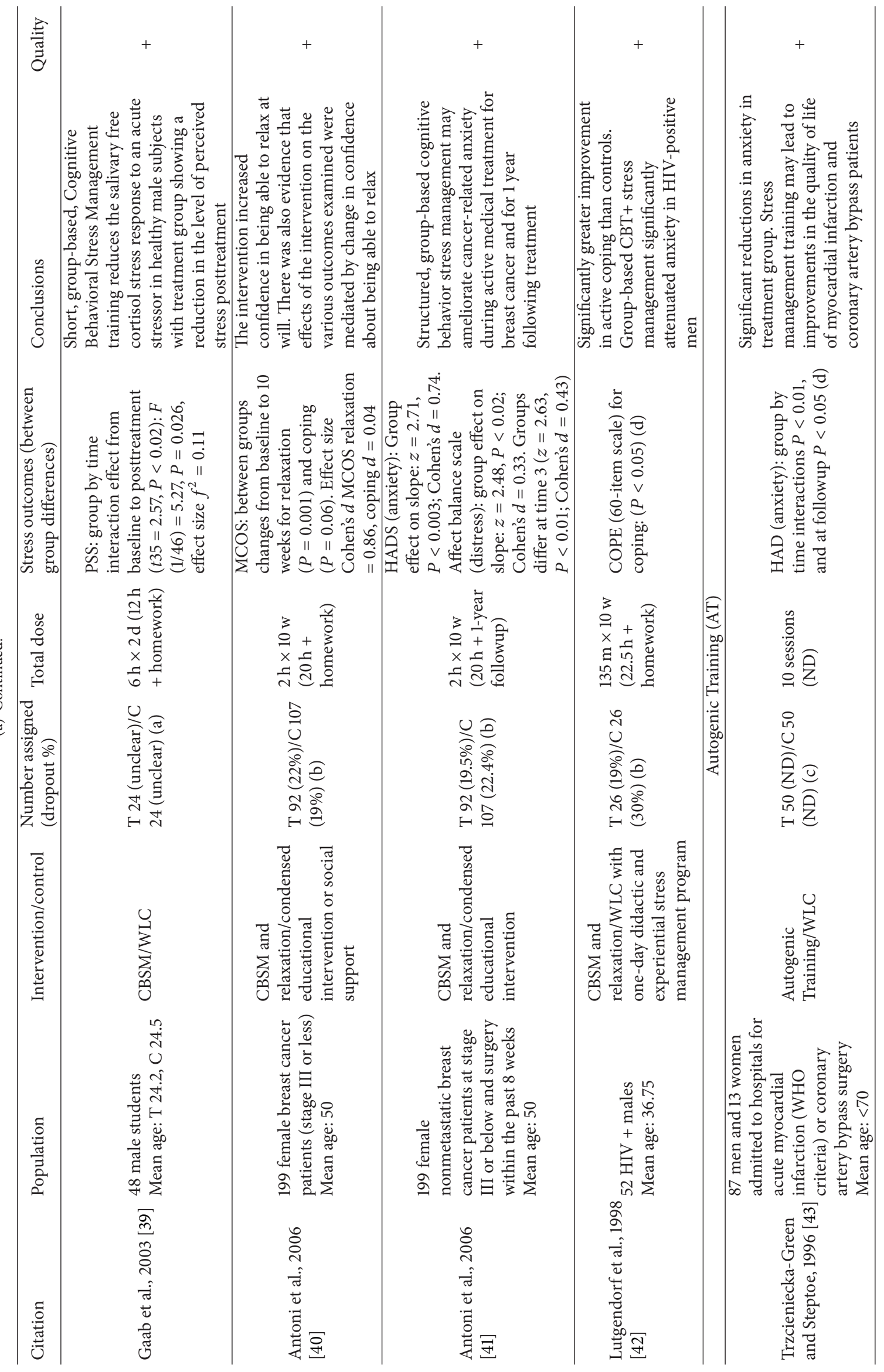




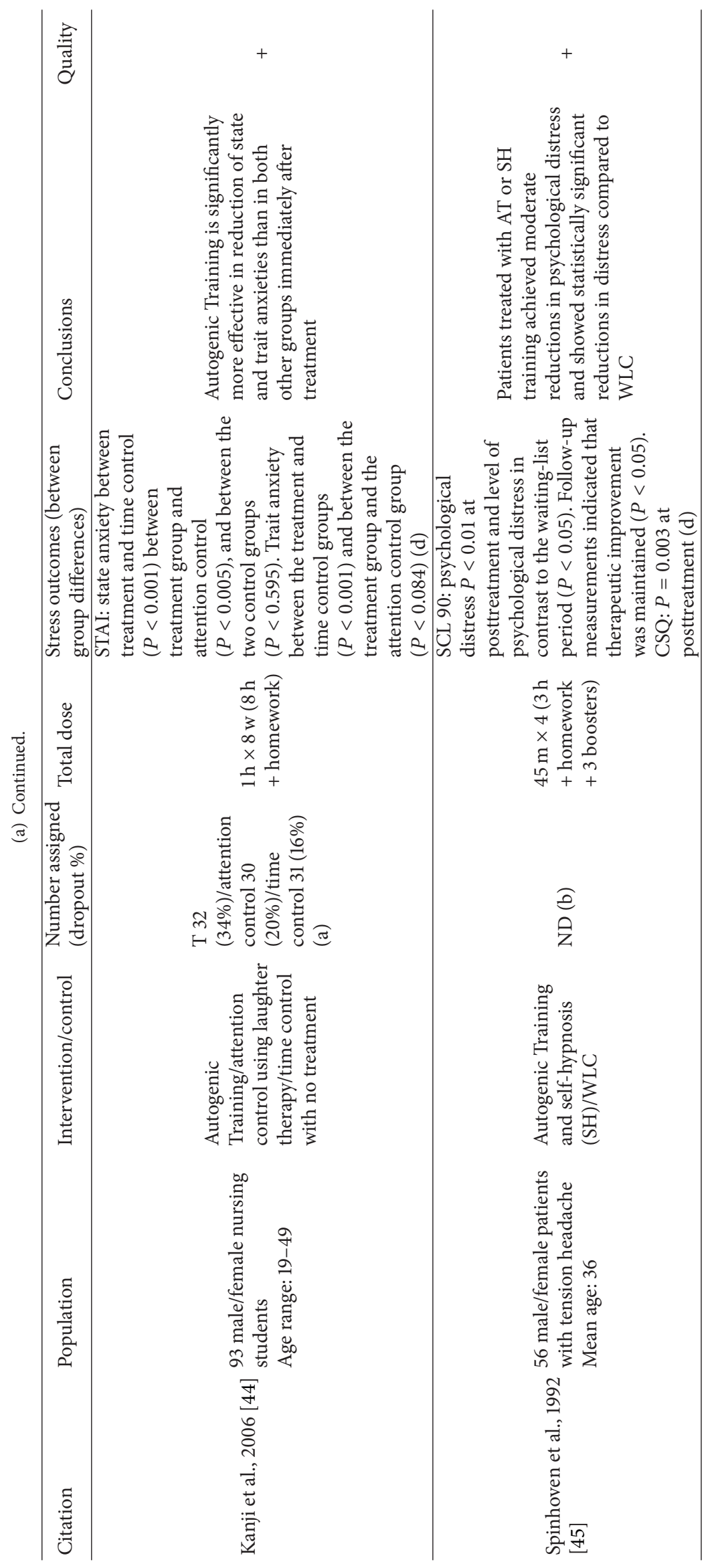




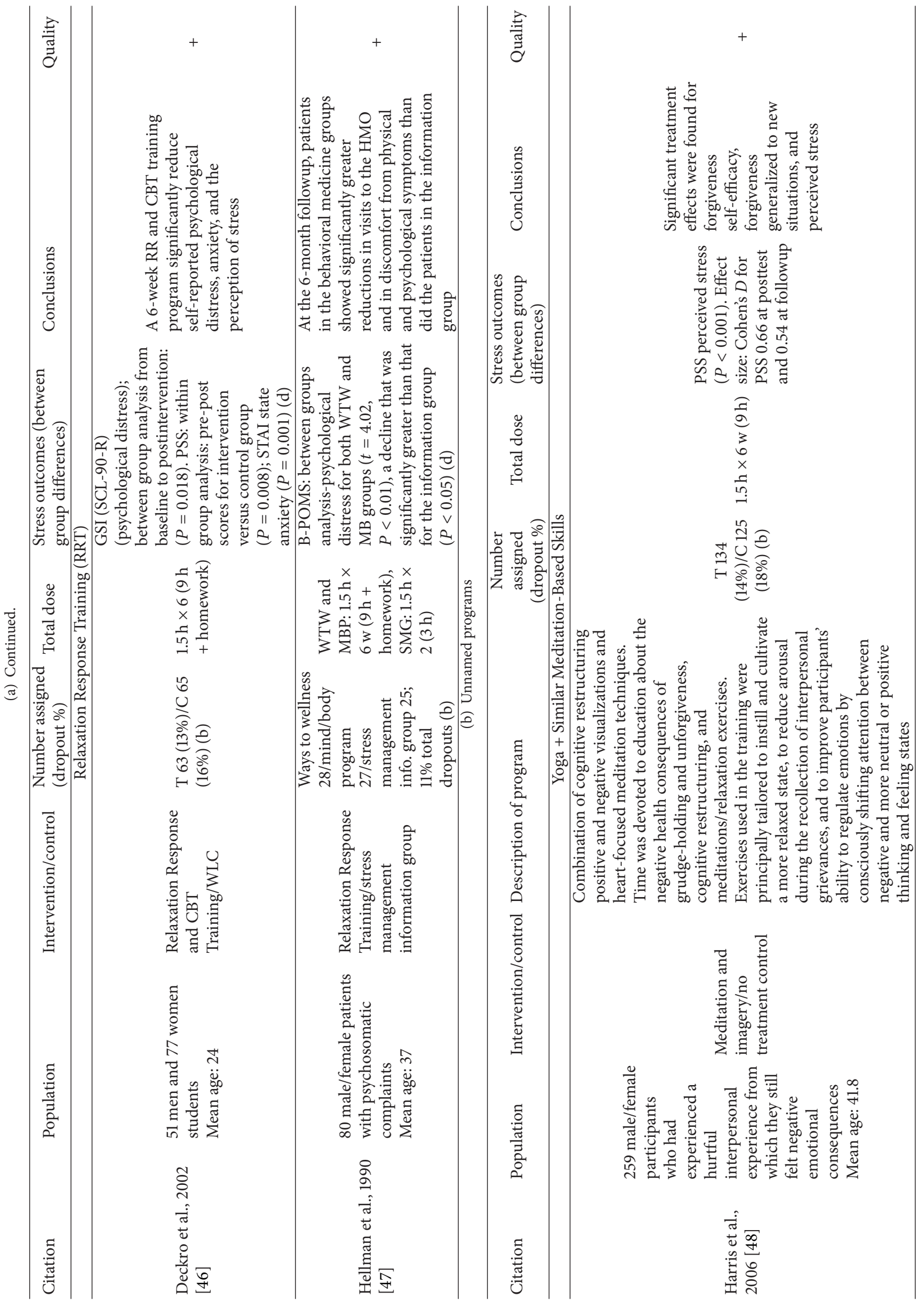




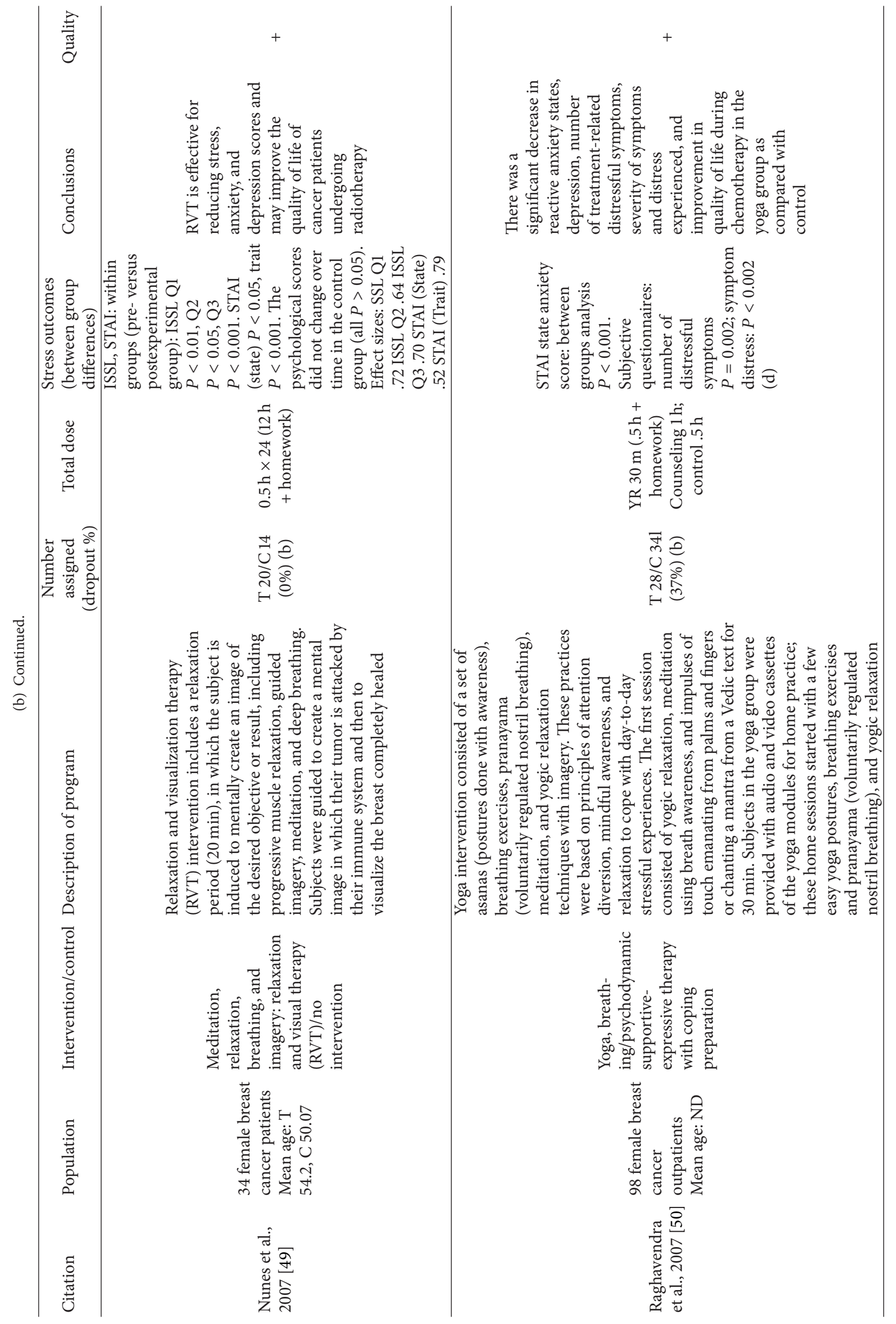




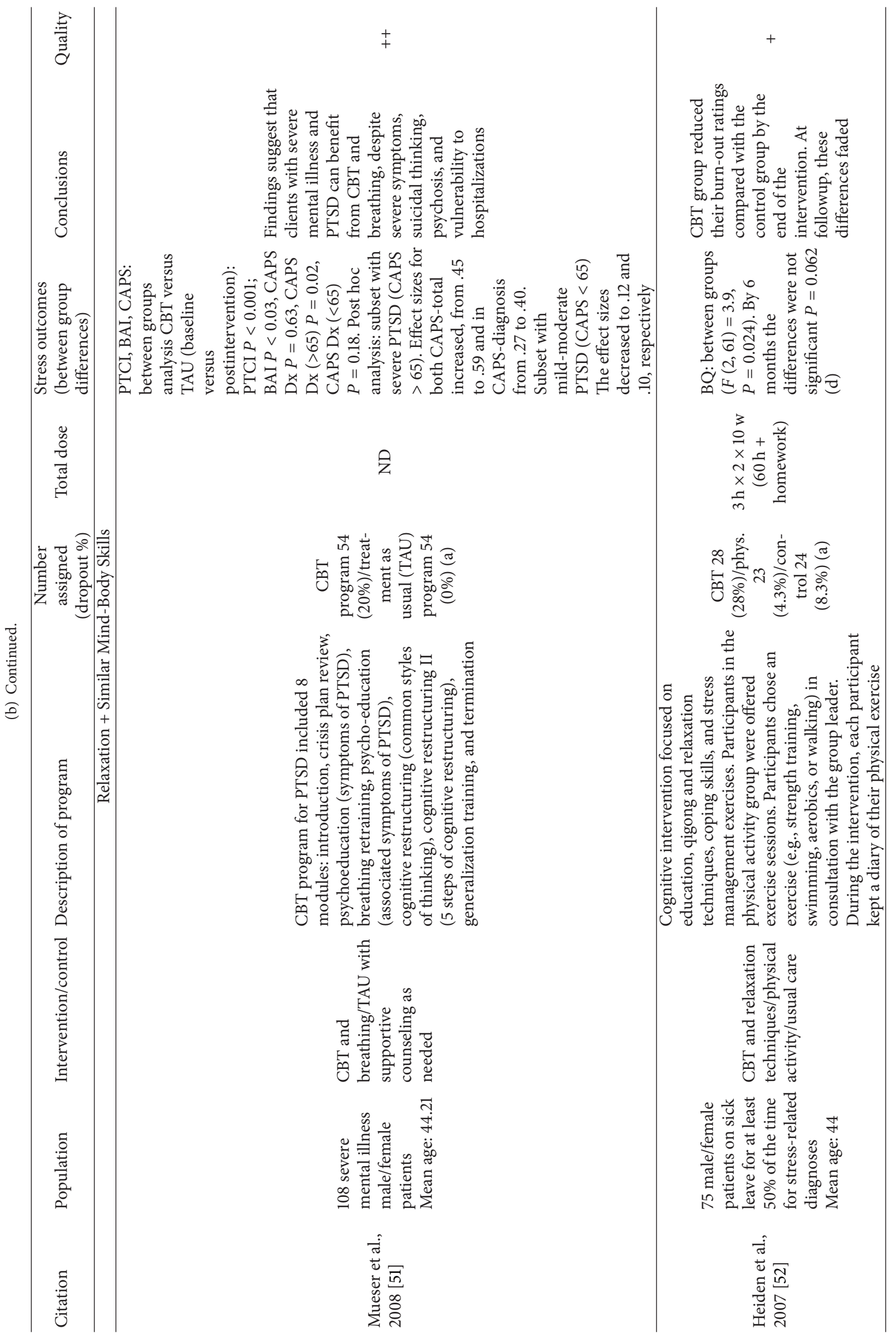



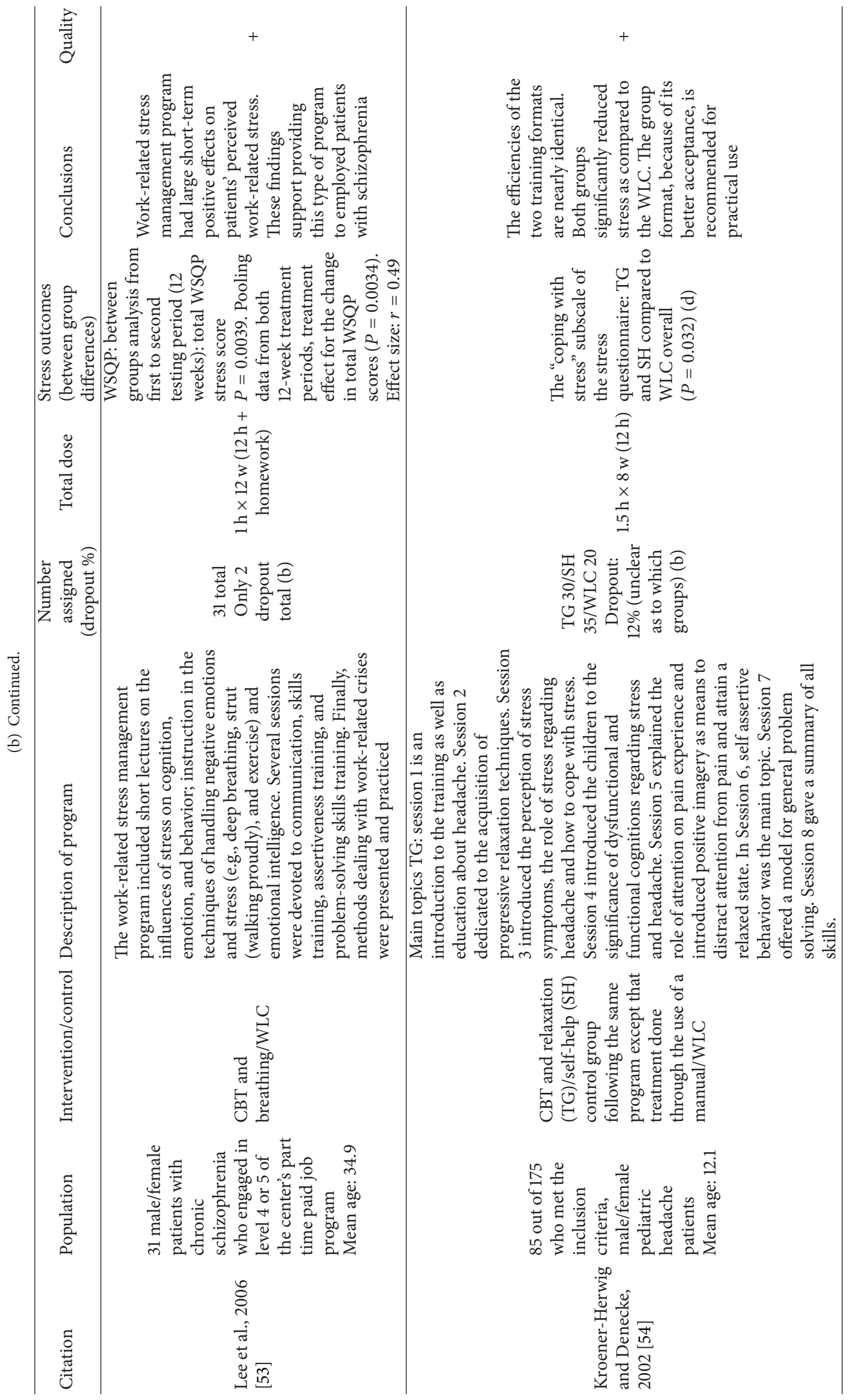


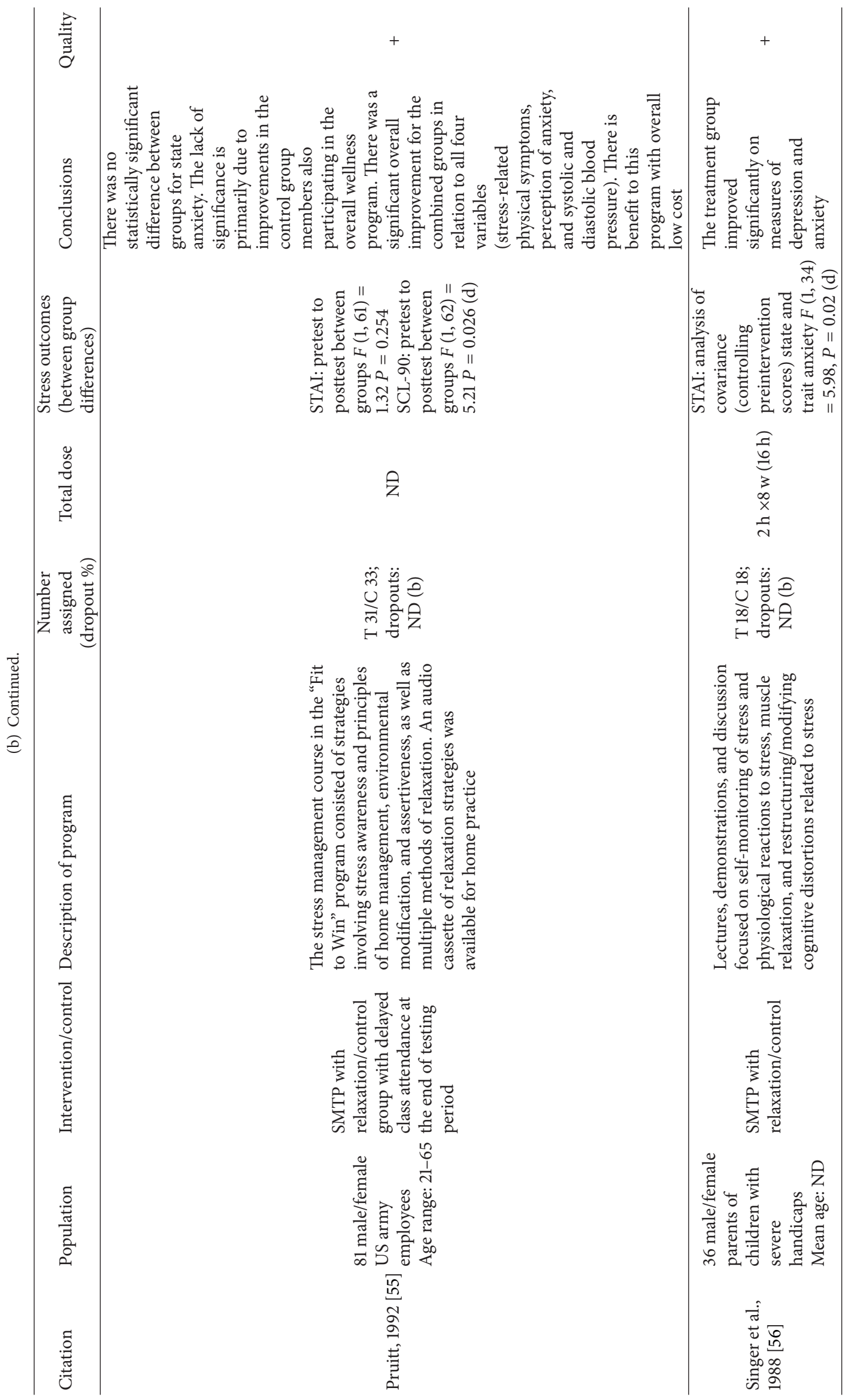




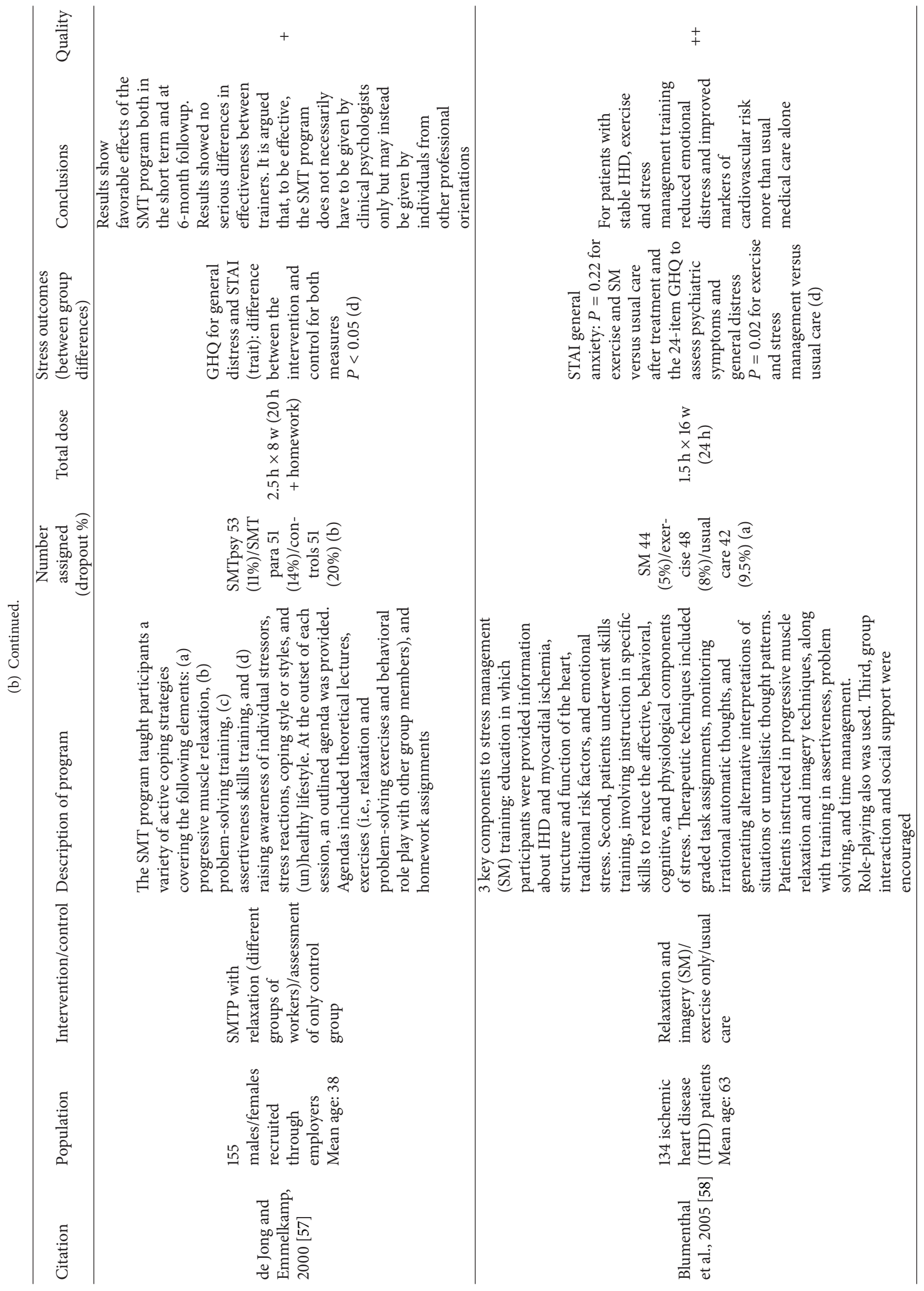




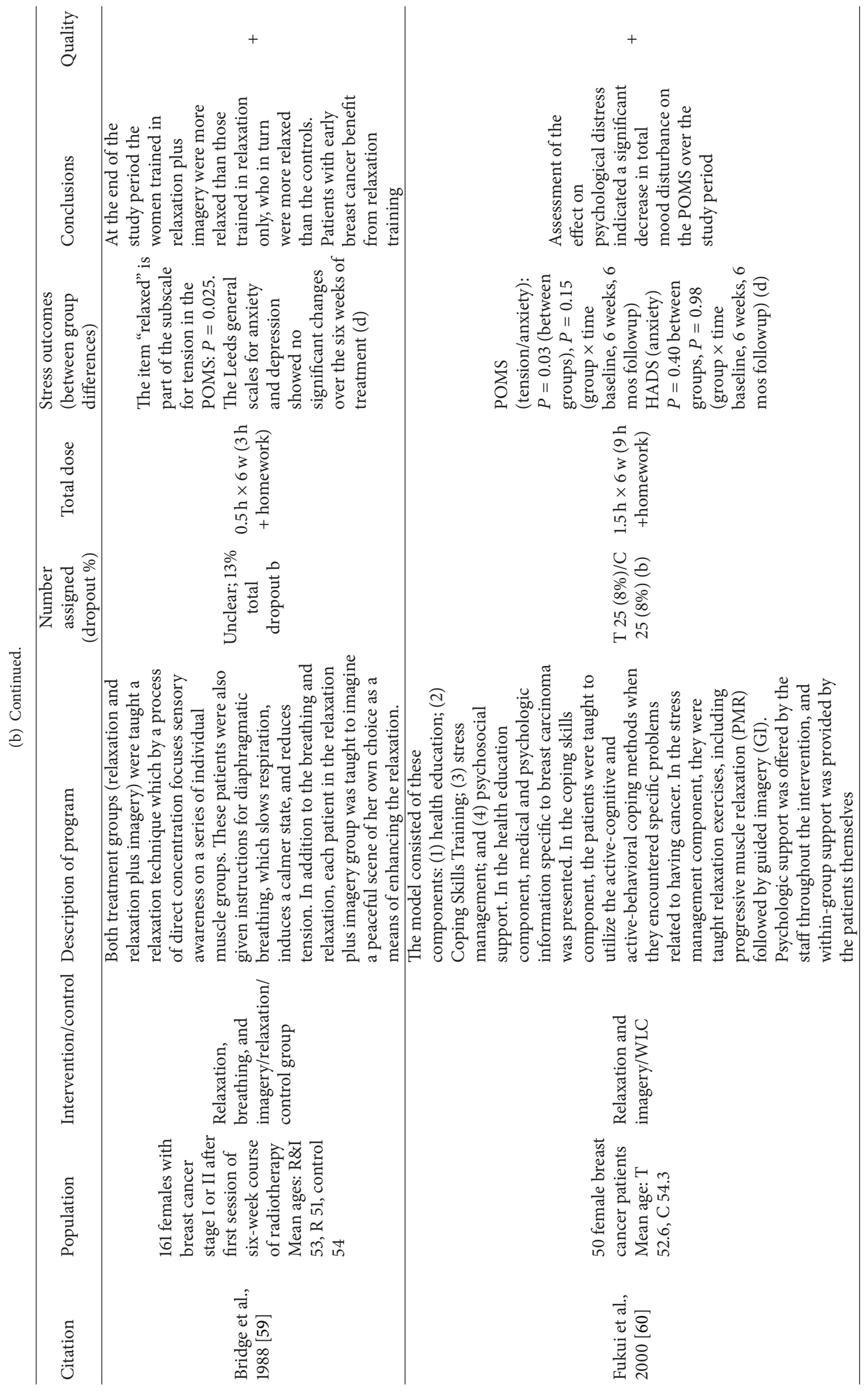




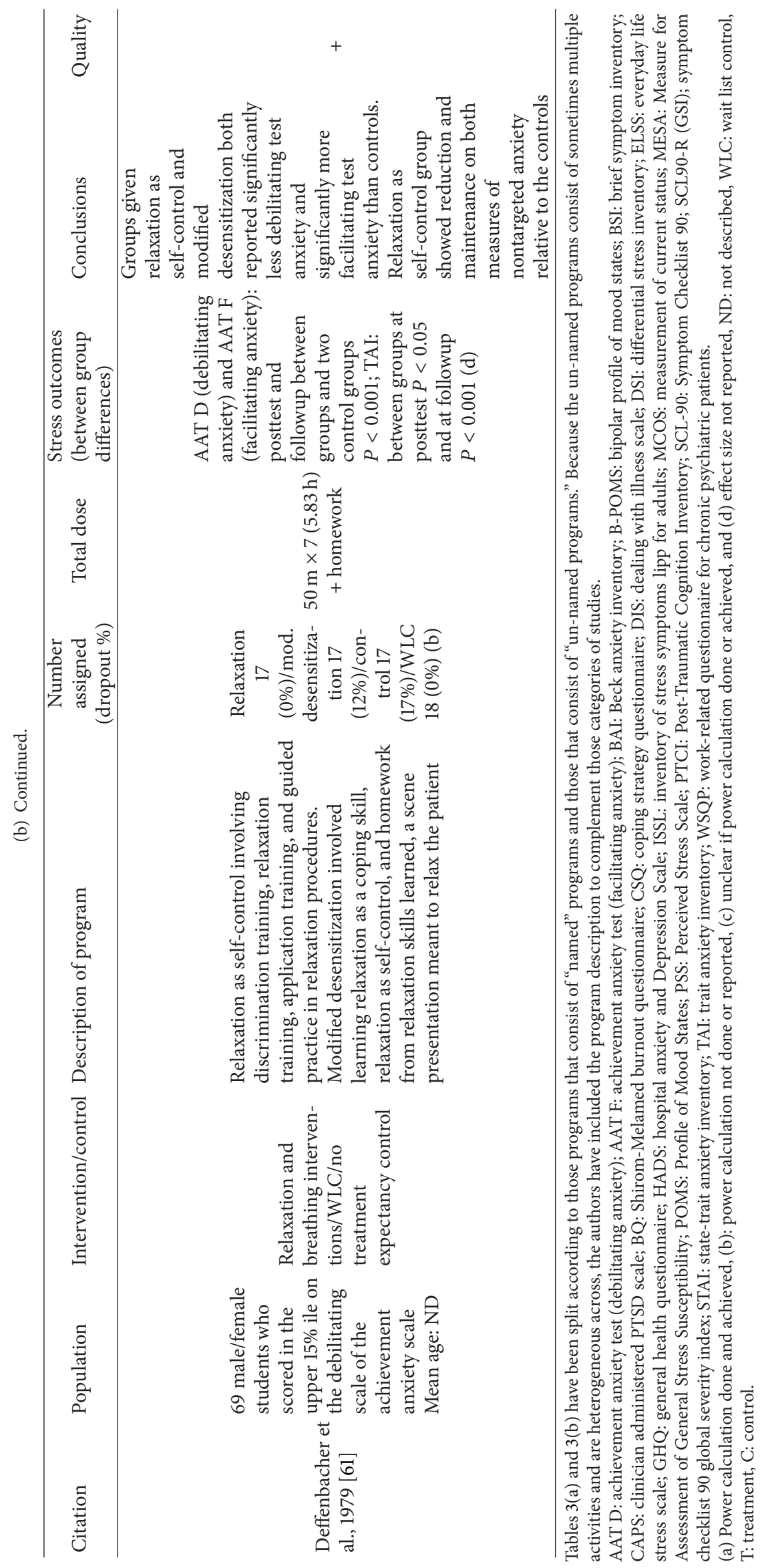


in significantly less burnout in 75 individuals with stressrelated conditions [52]. In the fourth study, 85 pediatric headache patients who completed a 12-hour CBT program incorporating progressive muscle relaxation had significant improvements in coping with stress [54]. Three studies characterized their intervention as an SMTP plus relaxation techniques. In one study of 81 US Army employees stationed at the Pentagon, an SMTP that combined multiple relaxation techniques resulted in significant reductions in distress but not anxiety [55]. A second study which implemented a 16hour SMTP with PMR in 36 parents of children with severe physical disabilities resulted in significant reductions in both state and trait anxiety [56]. A third study using a 20-hour SMTP program with PMR resulted in decreases in distress and trait anxiety in 155 police, hospital, and school employees [57].

Four-high quality studies implemented GI or breathing exercises with other relaxation techniques, and in each case the results were mixed: some stress-related outcome measures showed statistically significant differences, while others did not. In a study of 134 ischemic heart disease patients, a 24-hour program combining GI with Coping Skills Training and PMR resulted in significant reductions in distress but not in anxiety [58]. A second study implemented a ninehour program of GI with Coping Skills Training and PMR in 50 breast cancer patients and found a decrease in anxiety as measured by the Profile of Mood States (POMS) but not by the Hamilton Anxiety and Depression Scale (HADS) [60]. The third study used three hours of GI with breathing and relaxation exercises in 161 breast cancer patients undergoing radiotherapy; while tension scores on the POMS lessened significantly, the Leeds anxiety differences were nonsignificant [59]. The fourth study that used relaxation, breathing, and desensitization techniques in highly anxious psychology students produced statistically significant reductions in testtaking anxiety [61].

\subsection{Resource Requirements of Named Multimodal Programs.} In this section, the authors provide a subjective assessment of the resource requirements for these programs, based on the descriptive data collected: information describing the amount of time required for individual and practitioner or trainer involvement, facility and equipment needed, and estimated cost ranges. Because the unnamed programs were heterogeneous with regard to time for training and content involved in each session, the authors elected not to report on resource requirements for these. In determining what resources would potentially be required during the training phase for the named programs (see Figure 2(a)), the authors considered this as the period of time when a program was initially instituted and would require a trainer or instructor to teach self-management skills to participants. Since data was collected on the "dose" of the program training (i.e., the actual number of hours per week $\times$ number of weeks in which the program was delivered), the authors then categorized the amount of training time needed as minimal (less than 10 hours on average) or extensive (greater than 10 hours). Based on this information, the authors then estimated the amount of practitioner or trainer involvement required to teach these skills. Programs like MBSR and CBSM require substantially more specialized training of and sustained practitioner involvement, compared to interventions like AT and RRT which can be more quickly learned by participants. Using the descriptions of the intervention extracted from the data, the authors then codified facility requirements (i.e., an estimate of how much space is needed to learn the techniques), whether any equipment is necessary to learn the skills, and costs associated with the training (based on internet searches of the described programs). The authors present a similar assessment for the self-practice requirements (i.e., once the individual is fully trained and able to practice on his/her own) in Figure 2(b). Compared to conventional therapies, the resource requirements for both training and self-practice are all overall likely minimal. Once fully trained, service members should be able to practice these skills easily in any setting, with minimal time required, no equipment necessary, and at virtually no additional cost. While the main focus of this review was to report on the effectiveness of these multimodal programs in impacting emotional stress, the authors have additionally provided information about estimated resource requirements for military leadership and program managers in order to guide their decision making about the feasibility of integrating such programs into military settings. Whether these programs could be implemented "as is" or if they would need to be modified or adapted is not an assessment the authors have made, as only those in decision-making positions are able to definitively decide such feasibility issues.

\section{Discussion}

The programs described in this report have potential benefits for service members and their families. Since they primarily involve self-management skills, they can become self-empowering to the individual and can be used in any environment, with minimal time needed. This may be especially helpful to the population of individuals that are likely to refuse, delay, or feel stigmatized by conventional therapies. They are cost-effective strategies to prevent or manage stressors. Since they are multimodal, they may offer greater appeal than single-modality programs. There are very few to no adverse effects from these self-management skills when properly learned and practiced.

Although there are reviews in the literature to describe relevant programs that address specific issues (PTSD, resilience) in service member populations [64, 65], this comprehensive systematic review globally reviewed the literature on biopsychosocial multimodal programs, extracted the ones with high methodological quality and statistically significant reductions in stress (and similar keywords), and presented these results with estimated resource requirements. Because of this comprehensive approach, promising programs that have a strong evidence base, most of which were not evaluated in military populations, were able to be identified. This information is important for learning approaches that could be applied in these populations, especially since there is so 


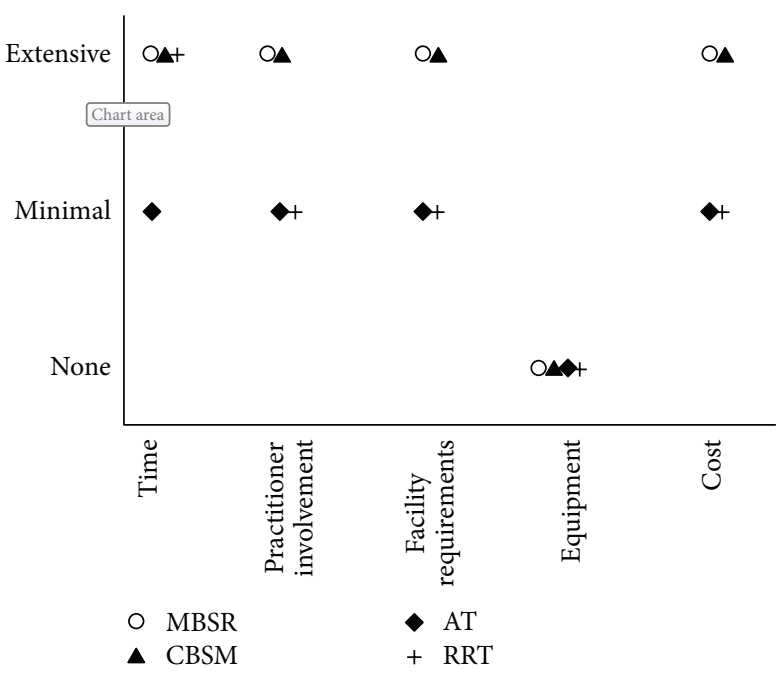

(a)

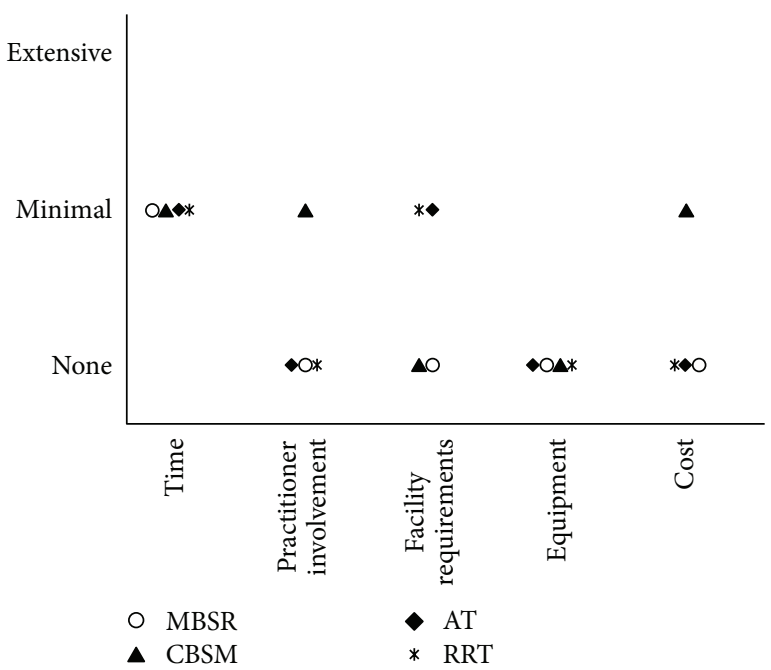

(b)

FIGURE 2: (a) Training requirements and (b) self-practice requirements.

little research on these topics in military populations. This data could potentially aid military leaders who are looking for evidence-based programs to reduce psychological stress and help guide their decision making about implementing these programs as described, or tailoring the needs of service members.

A fundamental problem associated with initial efforts to launch effective mind-body programs in practice is the limited evidence base to guide program choice. While evidencebased approaches may be desirable, such evidence is scarce. Even if evidence is available, the basic steps of program planning may lead conscientious planners to programs that have not been evaluated for their effectiveness. With this paper, the authors hope to stimulate thinking about translating this best evidence synthesis into practice, in order to make headway into the prevention and treatment of stress-related illness. The message to service members and their family members who are struggling with stress-related conditions is that they can change the way their body and mind react to stress by changing their thoughts, emotions, and behaviors. To the leadership, that is, understandably wary of making decisions without the proper evidentiary support, the authors offer the results of this comprehensive systematic review, demonstrating some promising directions, preliminary evidence of effectiveness for stress-related outcomes across all populations, and basic characterizations and descriptions of some of the self-care, skills training programs. The goals were to demystify them and to illustrate that many of them involve minimal cost and training time.

The US military success at shifting the combat focus from response to IED attacks in theater to better IED detection and armor to prevent injuries, termed "left of boom" [66] can serve as a powerful model for the mitigation of combat stress-related issues. A similar commitment to changing the paradigm from treatment of combat-related PTSD after it has been diagnosed to empowerment of troops and their families to take control over their physiologic and psychological responses to stress through skills training would represent a true shift to "left of PTSD" [67, 68].

3.1. Limitations of the Review. The authors of this comprehensive systematic review were only interested in assessing and reporting on the stress-related outcomes reported in the articles; whether they were primary or secondary outcomes was irrelevant to the review. Because of this, the authors did not paint the whole picture of each study captured and may have missed important elements of the original authors' intent. It was chosen to capture only those studies that reported on stress using the following terms: stress, anxiety, posttraumatic stress disorder, coping, resilience, hardiness, burnout, distress, or relaxation. The authors derived these terms by assessing the literature and consulting with subject matter experts. The authors acknowledge that this is most likely not a comprehensive and exhaustive list of all emotional stress-related terms, and the search may have missed pertinent studies that would have fit the inclusion criteria using other similarly related terms for stress.

Another limitation is that this review only included multimodal programs. The authors felt that these programs would include the dimensions of the BPS model and would have broader appeal by not focusing all efforts on one technique (e.g., yoga). As a result, any studies involving a single modality (i.e., only yoga, meditation, relaxation, or imagery) were excluded at the screening phase. The authors and colleagues are currently assessing the literature of singlemodal mind-body approaches in a more focused population relating to the military.

The authors only included those studies that have been published in the English language. While some systematic reviews consider the inclusion of only English-language studies as a limitation; doing so does not seriously compromise the outcome or implication for the majority of interventions and claims [69]. There has been some debate over this in the literature. The amount of effort and expense to include studies 
that have not been translated into the English language is a challenge to methodologists since the translator needs to be proficient in scientific language, able to understand the systematic review methodology, and be involved from the protocol development phase to clearly understand how to accurately code each review. The majority of systematic reviews, because of this challenge, only include the literature that is available in the English language.

The authors excluded all biofeedback studies during the review phase because it was decided that these were not truly self-management techniques; one would have to rely on the device during practice. Other programs, such as those learned through the internet or books, were excluded on this basis as well.

In this report, the authors have only described those studies that were of high quality and which reported statistically significant results between groups in controlled trial designs because of the interest in sharing only those that the authors felt confident in the estimate of the effect compared to a control group. Thus, excluded from this report were those programs that showed only within-group differences. Finally, quality assessment was based solely on internal validity criteria (the likelihood that the observed effects are due to bias) and did not take into account external validity (the likelihood that observed effects would occur outside the setting, i.e., generalizability), which is not usually assessed when evaluating quality in systematic review. Had the authors assessed external validity, the number of low-quality studies may have been decreased, allowing more studies to be shared. Future studies in the field should evaluate not only bias but also generalizability when assessing quality criteria.

\section{Conclusion}

The objective of this paper was to provide a descriptive overview and quantitative synthesis of information on multimodal programs that might be used for the self-management of emotional stress in our military communities and to consider this body of research as a guide to next steps in the research on implementation in military populations. MBSR, CBSM, AT, RRT, yoga plus similar meditation-based skills, and relaxation practices are the types of approaches emerging in the literature as the most promising for their benefits and ease of implementation in different settings. Implementing these identified training programs into military settings appears highly feasible, considering that resource requirements are minimal.

\section{Conflict of Interests}

None of the authors have any conflict of interests to report based on this project, including financial interests, consultant, institutional or other relationships that might lead to bias or a conflict of interests.

\section{Acknowledgments}

The authors would like to acknowledge Sasha Knowlton, MD, Matthew Fritts, MPH, PMP, E-424 RYT, and Viviane Enslein for their contributions to the project. Oral presentation was done by C. Crawford, D. Wallerstedt, and R. Khorsan. Biopsychosocial training programs for the self-management of emotional Stress: potential applications for the Military Armed Forces Health Protection Conference AFHPC, Hampton, VA, March 24, 2011. This project was funded by the US Army Medical Research Acquisition Activity, 820 Chandler Street, Fort Detrick, MD Award no. W81XWH08-1-0408 through Telemedicine and Advanced Technology Research Command (TATRC). The views, opinions, and/or findings contained in this report are those of the author(s) and should not be construed as an official department of the Army position, policy, or decision unless so designated by other documentation.

\section{References}

[1] J. Giordano and J. Walter, "Pain and psychopathology in military wounded: how etiology, epidemiology sustain an ethics of treatment," Practical Pain Management, vol. 7, no. 6, pp. 3442, 2007.

[2] P. Barnes, P. Powell-Griner, K. Mcfann, R. Nahin, United States Department of Health and Human Services, and Centers for Disease Control and Prevention, Advance Data From Vital and Health Statistics, Complementary and Alternative Medicine Use among Adults: United States, Hyattsville, Md, USA, 2002.

[3] F. McPherson and M. A. Schwenka, "Use of complementary and alternative therapies among active duty soldiers, military retirees, and family members at a military hospital," Military Medicine, vol. 169, no. 5, pp. 354-357, 2004.

[4] R. M. Bray, L. L. Hourani, K. L. R. Olmsted et al., "Department of Defense Survey of Health Related Behaviors among Active Duty Military Personnel," 2012, http://www.dtic.mil/cgi-bin/GetTRDoc?Location=U2\&doc=GetTRDoc.pdf\&AD=ADA465678.

[5] Klemm Analysis Group, Alternative Medicine Therapy: Assessment of Current Vha Practices and Opportunities, Washington, DC, USA, 1999.

[6] K. Kroesen, C. M. Baldwin, A. J. Brooks, and I. R. Bell, "US military veterans' perceptions of the conventional medical care system and their use of complementary and alternative medicine," Family Practice, vol. 19, no. 1, pp. 57-64, 2002.

[7] Healthcare Analysis \& Information Group (HAIG), Complementary and Alternative Medicine, Washington, DC, USA, 2002.

[8] G. L. Engel, "The need for a new medical model: a challenge for biomedicine," Science, vol. 196, no. 4286, pp. 129-136, 1977.

[9] M. Havelka, J. D. Lučanin, and D. Lučanin, "Biopsychosocial model-the integrated approach to health and disease," Collegium Antropologicum, vol. 33, no. 1, pp. 303-310, 2009.

[10] The Center for Mind-Body Medicine, "Healing the Wounds of War Successes," http://www.jamesgordonmd.com/z_pdfs/ CMBM_Groundbreaking_Global_Work.pdf.

[11] Benson-Henry Institute for Mind Body Medicine, "Programs andServices," http://www.mbmi.org/home.

[12] Center of Timeless Being and Integrative Restoration Irest, "Integrative Restoration Irest," http://www.nondual.com/ events/pdf/10/iRest\%20Brochure\%20Generic.pdf.

[13] Institute of Heartmath, "Heartmath Military Warrior Initiative," http://www.heartmath.org/joinorgive/heartmath_military_ warrior_initiative.html. 
[14] Magis Group, "Who We Are," http://magisgroup.com/.

[15] G. H. Guyatt, A. D. Oxman, G. E. Vist, R. Kunz, Y. Falck-Ytter, and H. J. Schünemann, "What is "quality of evidence" and why is it important to clinicians?" British Medical Journal, vol. 336, no. 7651, pp. 995-998, 2008.

[16] A. D. Oxman and G. H. Guyatt, "Guidelines for reading literature reviews," Canadian Medical Association Journal, vol. 138, no. 8, pp. 697-703, 1988.

[17] S. I. G. Network, Sign 50: A Guideline Developer's Handbook, 2010.

[18] A. Chiesa and A. Serretti, "Mindfulness-based stress reduction for stress management in healthy people: a review and metaanalysis," Journal of Alternative and Complementary Medicine, vol. 15, no. 5, pp. 593-600, 2009.

[19] P. Grossman, L. Niemann, S. Schmidt, and H. Walach, "Mindfulness-based stress reduction and health benefits: a meta-analysis," Journal of Psychosomatic Research, vol. 57, no. 1, pp. 35-43, 2004.

[20] J. L. Brown and P. A. Vanable, "Cognitive-behavioral stress management interventions for persons living with HIV: a review and critique of the literature," Annals of Behavioral Medicine, vol. 35, no. 1, pp. 26-40, 2008.

[21] M. H. Antoni, S. K. Lutgendorf, B. Blomberg et al., "Cognitivebehavioral stress management reverses anxiety-related leukocyte transcriptional dynamics," Biological Psychiatry, vol. 71, no. 4, pp. 366-372, 2012.

[22] N. Kanji, A. R. White, and E. Ernst, "Autogenic training for tension type headaches: a systematic review of controlled trials," Complementary Therapies in Medicine, vol. 14, no. 2, pp. 144-150, 2006.

[23] A. L. Hassett and R. N. Gevirtz, "Nonpharmacologic treatment for fibromyalgia: patient education, cognitive-behavioral therapy, relaxation techniques, and complementary and alternative medicine," Rheumatic Disease Clinics of North America, vol. 35, no. 2, pp. 393-407, 2009.

[24] W. Luthe and J. Schultz, Autogenic Therapy, British Autogenic Society, New York, NY, USA, 2001.

[25] S. Jain, S. L. Shapiro, S. Swanick et al., "A randomized controlled trial of mindfulness meditation versus relaxation training: effects on distress, positive states of mind, rumination, and distraction," Annals of Behavioral Medicine, vol. 33, no. 1, pp. 1121, 2007.

[26] K. A. Williams, M. M. Kolar, B. E. Reger, and J. C. Pearson, "Evaluation of a wellness-based mindfulness stress reduction intervention: a controlled trial," American Journal of Health Promotion, vol. 15, no. 6, pp. 422-432, 2001.

[27] H. Benson, J. F. Beary, and M. P. Carol, "The relaxation response," Psychiatry, vol. 37, no. 1, pp. 37-46, 1974.

[28] J. A. Dusek, B.-H. Chang, J. Zaki et al., "Association between oxygen consumption and nitric oxide production during the relaxation response," Medical Science Monitor, vol. 12, no. 1, pp. CR1-CR10, 2006.

[29] H. Benson, J. B. Kotch, and K. D. Crassweller, "Stress and hypertension: interrelations and management," Cardiovascular Clinics, vol. 9, no. 1, pp. 113-124, 1978.

[30] E. K. Pradhan, M. Baumgarten, P. Langenberg et al., "Effect of mindfulness-based stress reduction in rheumatoid arthritis patients," Arthritis Care and Research, vol. 57, no. 7, pp. 1134$1142,2007$.

[31] A. M. Tacón, J. McComb, Y. Caldera, and P. Randolph, "Mindfulness meditation, anxiety reduction, and heart disease: a pilot study," Family \& Community Health, vol. 26, no. 1, pp. 25-33, 2003.

[32] S. L. Shapiro, G. E. Schwartz, and G. Bonner, "Effects of mindfulness-based stress reduction on medical and premedical students," Journal of Behavioral Medicine, vol. 21, no. 6, pp. 581599, 1998.

[33] D. Oman, S. L. Shapiro, C. E. Thoresen, T. G. Plante, and T. Flinders, "Meditation lowers stress and supports forgiveness among college students: a randomized controlled trial," Journal of American College Health, vol. 56, no. 5, pp. 569-578, 2008.

[34] M. Speca, L. E. Carlson, E. Goodey, and M. Angen, "A randomized, wait-list controlled clinical trial: the effect of a mindfulness meditation-based stress reduction program on mood and symptoms of stress in cancer outpatients," Psychosomatic Medicine, vol. 62, no. 5, pp. 613-622, 2000.

[35] N. L. McCain, D. P. Gray, R. K. Elswick Jr. et al., "A randomized clinical trial of alternative stress management interventions in persons with HIV infection," Journal of Consulting and Clinical Psychology, vol. 76, no. 3, pp. 431-441, 2008.

[36] M. Claesson, L. S. Birgander, B. Lindahl et al., "Women's hearts - stress management for women with ischemic heart disease: explanatory analyses of a randomized controlled trial," Journal of Cardiopulmonary Rehabilitation, vol. 25, no. 2, pp. 93-102, 2005.

[37] S. Berger, T. Schad, V. Von Wyl et al., "Effects of cognitive behavioral stress management on HIV-1 RNA, CD4 cell counts and psychosocial parameters of HIV-infected persons," AIDS, vol. 22, no. 6, pp. 767-775, 2008.

[38] J. Gaab, L. Sonderegger, S. Scherrer, and U. Ehlert, "Psychoneuroendocrine effects of cognitive-behavioral stress management in a naturalistic setting - a randomized controlled trial," Psychoneuroendocrinology, vol. 31, no. 4, pp. 428-438, 2006.

[39] J. Gaab, N. Blättler, T. Menzi, B. Pabst, S. Stoyer, and U. Ehlert, "Randomized controlled evaluation of the effects of cognitivebehavioral stress management on cortisol responses to acute stress in healthy subjects," Psychoneuroendocrinology, vol. 28, no. 6, pp. 767-779, 2003.

[40] M. H. Antoni, S. C. Lechner, A. Kazi et al., "How stress management improves quality of life after treatment for breast cancer," Journal of Consulting and Clinical Psychology, vol. 74, no. 6, pp. 1143-1152, 2006.

[41] M. H. Antoni, S. R. Wimberly, S. C. Lechner et al., "Reduction of cancer-specific thought intrusions and anxiety symptoms with a stress management intervention among women undergoing treatment for breast cancer," American Journal of Psychiatry, vol. 163, no. 10, pp. 1791-1797, 2006.

[42] S. K. Lutgendorf, M. H. Antoni, G. Ironson et al., "Changes in cognitive coping skills and social support during cognitive behavioral stress management intervention and distress outcomes in symptomatic human immunodeficiency virus (HIV)seropositive gay men," Psychosomatic Medicine, vol. 60, no. 2, pp. 204-214, 1998.

[43] A. Trzcieniecka-Green and A. Steptoe, "The effects of stress management on the quality of life of patients following acute myocardial infarction or coronary bypass surgery," European Heart Journal, vol. 17, no. 11, pp. 1663-1670, 1996.

[44] N. Kanji, A. White, and E. Ernst, "Autogenic training to reduce anxiety in nursing students: randomized controlled trial," Journal of Advanced Nursing, vol. 53, no. 6, pp. 729-735, 2006.

[45] P. Spinhoven, A. C. G. Linssen, R. Van Dyck, and F. G. Zitman, "Autogenic training and self-hypnosis in the control of tension 
headache," General Hospital Psychiatry, vol. 14, no. 6, pp. 408415, 1992.

[46] G. R. Deckro, K. M. Ballinger, M. Hoyt et al., “The evaluation of a mind/body intervention to reduce psychological distress and perceived stress in college students," Journal of American College Health, vol. 50, no. 6, pp. 281-287, 2002.

[47] C. J. C. Hellman, M. Budd, J. Borysenko, D. C. McClelland, and H. Benson, "A study of the effectiveness of two group behavioral medicine interventions for patients with psychosomatic complaints," Behavioral Medicine, vol. 16, no. 4, pp. 165-173, 1990.

[48] A. H. S. Harris, F. Luskin, S. B. Norman et al., "Effects of a group forgiveness intervention on forgiveness, perceived stress, and trait-anger," Journal of Clinical Psychology, vol. 62, no. 6, pp. 715733, 2006.

[49] D. F. T. Nunes, A. L. Rodriguez, F. da Silva Hoffmann et al., "Relaxation and guided imagery program in patients with breast cancer undergoing radiotherapy is not associated with neuroimmunomodulatory effects," Journal of Psychosomatic Research, vol. 63, no. 6, pp. 647-655, 2007.

[50] R. M. Raghavendra, R. Nagarathna, H. R. Nagendra et al., "Effects of an integrated yoga programme on chemotherapyinduced nausea and emesis in breast cancer patients," European Journal of Cancer Care, vol. 16, no. 6, pp. 462-474, 2007.

[51] K. T. Mueser, S. D. Rosenberg, H. Xie et al., "A randomized controlled trial of cognitive-behavioral treatment for posttraumatic stress disorder in severe mental illness," Journal of Consulting and Clinical Psychology, vol. 76, no. 2, pp. 259-271, 2008.

[52] M. Heiden, E. Lyskov, M. Nakata, K. Sahlin, T. Sahlin, and M. Barnekow-Bergkvist, "Evaluation of cognitive behavioural training and physical activity for patients with stress-related illnesses: a randomized controlled study," Journal of Rehabilitation Medicine, vol. 39, no. 5, pp. 366-373, 2007.

[53] H.-L. Lee, H. K.-L. Tan, H.-I. Ma, C.-Y. Tsai, and Y.-K. Liu, "Effectiveness of a work-related stress management program in patients with chronic schizophrenia," American Journal of Occupational Therapy, vol. 60, no. 4, pp. 435-441, 2006.

[54] B. Kroener-Herwig and H. Denecke, "Cognitive-behavioral therapy of pediatric headache: are there differences in efficacy between a therapist-administered group training and a self-help format?" Journal of Psychosomatic Research, vol. 53, no. 6, pp. 1107-1114, 2002.

[55] R. H. Pruitt, "Effectiveness and cost efficiency of interventions in health promotion," Journal of Advanced Nursing, vol. 17, no. 8, pp. 926-932, 1992.

[56] G. H. S. Singer, L. K. Irvin, and N. Hawkins, "Stress management training for parents of children with severe handicaps," Mental Retardation, vol. 26, no. 5, pp. 269-277, 1988.

[57] G. M. de Jong and P. M. Emmelkamp, "Implementing a stress management training: comparative trainer effectiveness," Journal of Occupational Health Psychology, vol. 5, no. 2, pp. 309$320,2000$.

[58] J. A. Blumenthal, A. Sherwood, M. A. Babyak et al., "Effects of exercise and stress management training on markers of cardiovascular risk in patients with ischemic heart disease: a randomized controlled trial," Journal of the American Medical Association, vol. 293, no. 13, pp. 1626-1634, 2005.

[59] L. R. Bridge, P. Benson, P. C. Pietroni, and R. G. Priest, "Relaxation and imagery in the treatment of breast cancer," British Medical Journal, vol. 297, no. 6657, pp. 1169-1172, 1988.

[60] S. Fukui, A. Kugaya, H. Okamura et al., "A psychosocial group intervention for Japanese women with primary breast carcinoma," Cancer, vol. 89, no. 5, pp. 1026-1036, 2000.
[61] J. L. Deffenbacher, H. Mathis, and A. C. Michaels, "Two selfcontrol procedures in the reduction of targeted and nontargeted anxieties," Journal of Counseling Psychology, vol. 26, no. 2, pp. 120-127, 1979.

[62] C.-Y. Feng, H. Chu, C.-H. Chen et al., "The effect of cognitive behavioral group therapy for depression: a meta-analysis 20002010," Worldviews on Evidence-Based Nursing, vol. 9, no. 1, pp. 2-17, 2012.

[63] R. L. Gould, M. C. Coulson, and R. J. Howard, "Efficacy of cognitive behavioral therapy for anxiety disorders in older people: a meta-analysis and meta-regression of randomized controlled trials," Journal of the American Geriatrics Society, vol. 60, no. 2, pp. 218-229, 2012.

[64] L. L. Hourani, C. L. Council, R. C. Hubal, and L. B. Strange, "Approaches to the primary prevention of posttraumatic stress disorder in the military: a review of the stress control literature," Military Medicine, vol. 176, no. 7, pp. 721-730, 2011.

[65] B. J. Morgan and S. C. Garmon Bibb, "Assessment of military population-based psychological resilience programs," Military Medicine, vol. 176, no. 9, pp. 976-985, 2011.

[66] R. Atkinson, "Left of boom: the struggle to defeat roadside bombs," in The Washington Post, 2006.

[67] Department of Defense, The Post-Deployment Health Reassessment, "Force Health Protection and Readiness Policy and Programs. Battlemind Training," http://fhp.osd.mil/pdhrainfo/ battlemind.jsp.

[68] AUSA News, "Clinic Expands Scope to Meet New Need," 2012, http://www3.ausa.org/webpub/DeptAUSANews.nsf/byid/ PGRH-6RUHNQ.

[69] D. Moher, P. Pham, T. P. Klassen et al., "What contributions do languages other than English make on the results of metaanalyses?" Journal of Clinical Epidemiology, vol. 53, no. 9, pp. 964-972, 2000. 


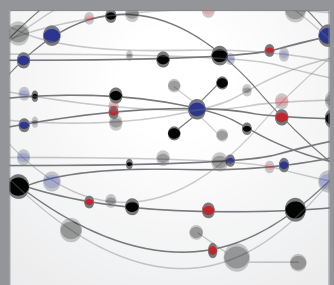

The Scientific World Journal
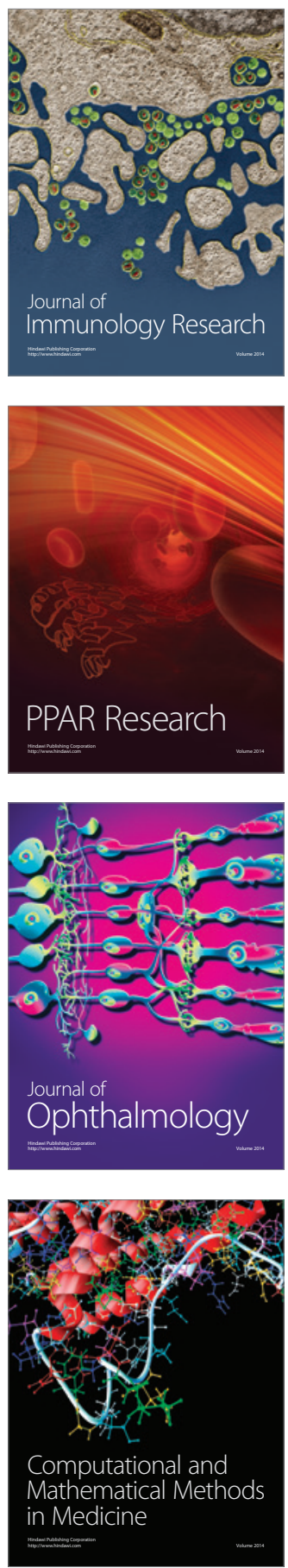

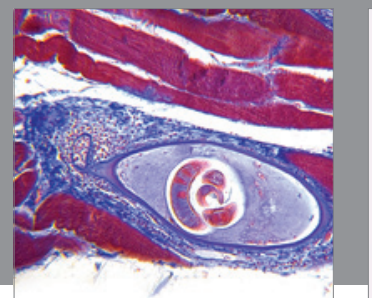

Gastroenterology

Research and Practice
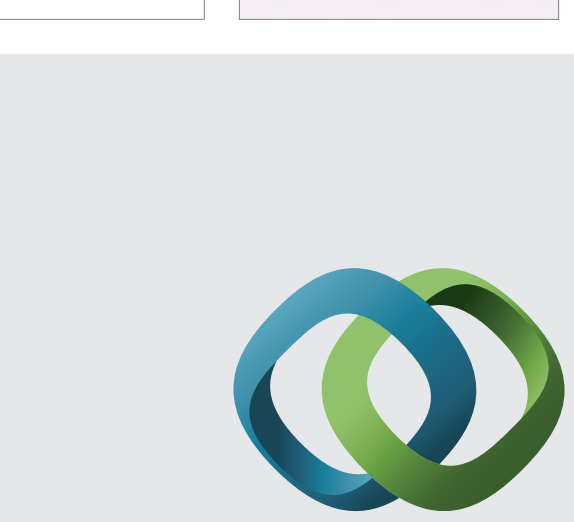

\section{Hindawi}

Submit your manuscripts at

http://www.hindawi.com
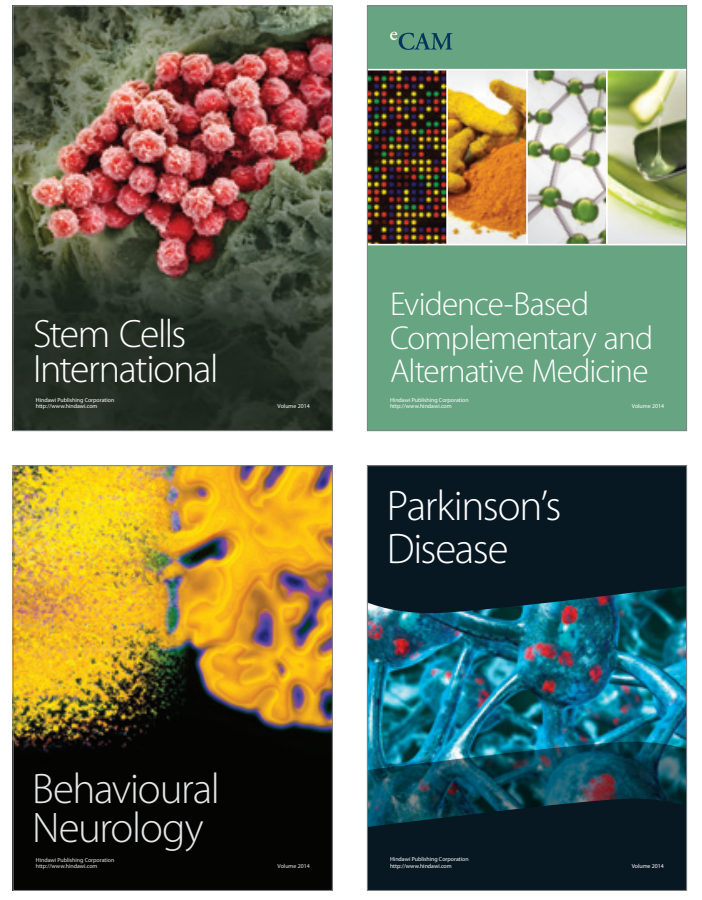
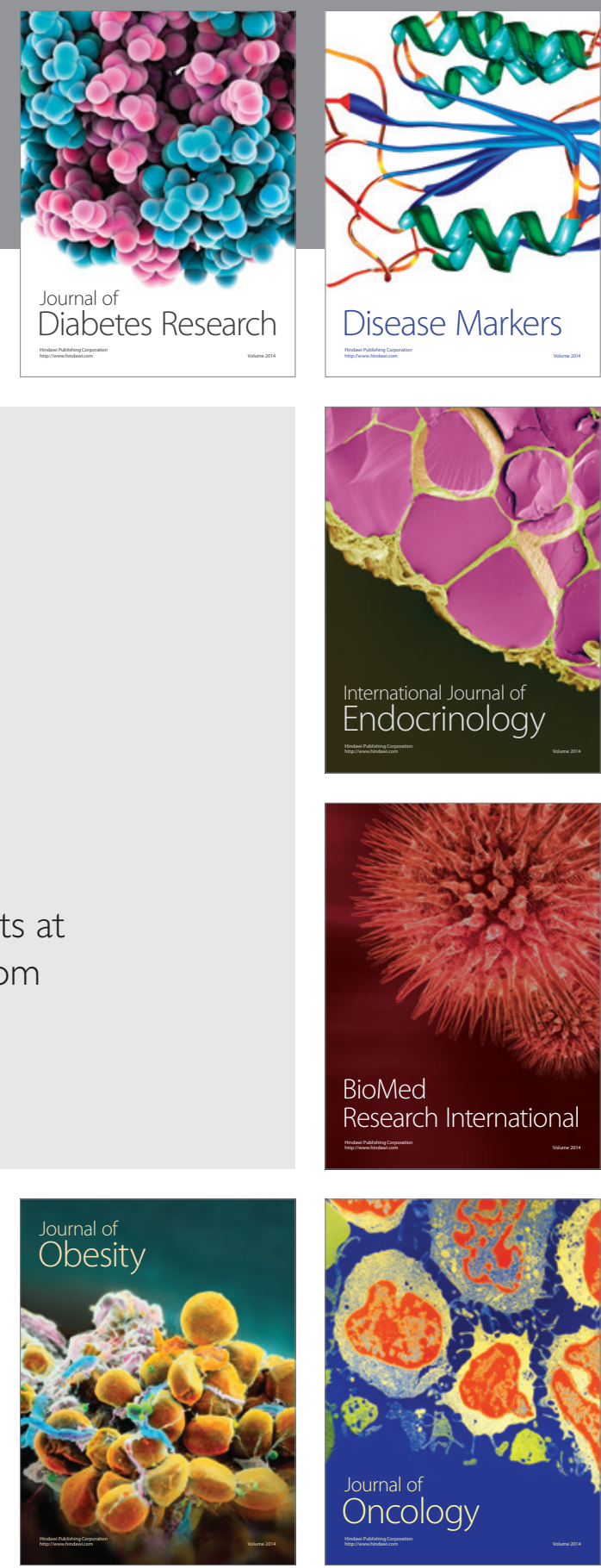

Disease Markers
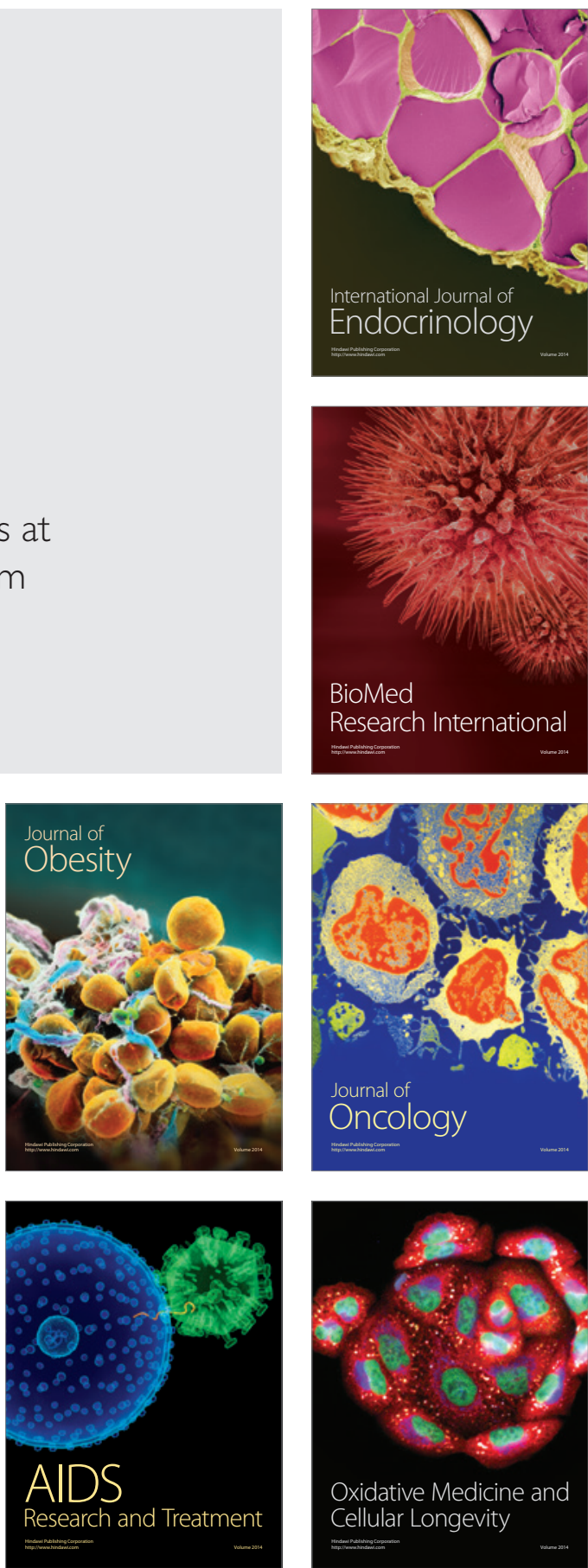